\title{
Investigating Cold Dust Properties of 12 Nearby Dwarf Irregular Galaxies by Hierarchical Bayesian Spectral Energy Distribution Fitting
}

\author{
Zhengxue Chang ${ }^{1,2}$ (1), Jianjun Zhou ${ }^{1,3}$ (1), Isabella Lamperti ${ }^{4,5,6}$ (D) Amélie Saintongel ${ }^{4}$, Jarken Esimbek ${ }^{1,3}$, Dalei Li $^{1,3}$, \\ Yuxin $\mathrm{He}^{1,3}$ (1) , Jianjie Qiu ${ }^{7}$ (10), Jun $\mathrm{Li}^{1,2}$, Zeming Zhou ${ }^{8}$, Xindi Tang ${ }^{1,3}$ (1) , Gang Wu ${ }^{9}$ (1) Weiguang $\mathrm{Ji}^{1}$, Mengke Zhao ${ }^{1,2}$, and \\ Minhua Zhou $^{10}$ (D) \\ ${ }^{1}$ Xinjiang Astronomical Observatory, Chinese Academy of Sciences, 150 Science 1-Street, Urumqi, Xinjiang 830011, People’s Republic of China; zhoujj@xao.ac.cn \\ ${ }^{2}$ University of Chinese Academy of Sciences, No.19A Yuquan Road, Shijingshan District, Beijing, 100049, People's Republic of China \\ ${ }^{3}$ Key Laboratory of Radio Astronomy, Chinese Academy of Sciences 150 Science 1-Street, Urumqi, Xinjiang 830011, People's Republic of China \\ ${ }^{4}$ Department of Physics and Astronomy, University College London, Gower Street, London WC1E 6BT, UK; isabellalamperti@gmail.com \\ ${ }^{5}$ European Southern Observatory, Karl-Schwarzschild-Strasse 2, D-85748 Garching bei München, Germany \\ ${ }^{6}$ Centro de Astrobiología (CSIC-INTA), Ctra. de Ajalvir, Km 4, E-28850, Torrejón de Ardoz, Madrid, Spain \\ ${ }^{7}$ School of Physics and Astronomy, Sun Yat-sen University, 2 Da Xue Road, Tangjia, Zhuhai 519000, Guangdong Province, People's Republic of China \\ ${ }^{8}$ Department of Astronomy, Beijing Normal University, Beijing, 100875, People's Republic of China \\ ${ }^{9}$ Max-Planck-Institut für Radioastronomie, Auf dem Hügel 69, D-53121 Bonn, Germany \\ ${ }^{10}$ School of Physics and Electronic Information, Shangrao Normal University, 401 Zhimin Road, Shangrao 334001, People's Republic of China \\ Received 2021 March 9; revised 2021 May 3; accepted 2021 May 4; published 2021 July 5
}

\begin{abstract}
Combining infrared and submillimeter observations and applying a two-temperature modified blackbody (TMBB) model with a hierarchical Bayesian technique, we model the spectral energy distribution of 12 nearby dwarf irregular (dIrr) galaxies. We aim to probe potential submillimeter excess emission at 350, 500, and $850 \mu \mathrm{m}$ and investigate the properties of cold dust parameters. Based on the TMBB model with cold dust emissivity index $\left(\beta_{\mathrm{c}}\right)$ fixed to 2, one galaxy shows $500 \mu \mathrm{m}$ excess emission and nine galaxies show excess at $850 \mu \mathrm{m}$ (five of them still show $850 \mu \mathrm{m}$ excess in the case of free $\beta_{\mathrm{c}}$ ). We find that the $850 \mu \mathrm{m}$ excess emission is easily detected in the dIrr galaxies with low star formation activity. The $850 \mu \mathrm{m}$ excess is more frequent and more prominent in dIrr galaxies with low molecular hydrogen gas mass fraction or low ratios between cold dust mass and gas mass. As galaxies evolve, the ratios between atomic hydrogen gas mass and stellar mass decrease and the $850 \mu \mathrm{m}$ excess emission tends to decrease or even disappear. Our results suggest that the cold dust temperature may increase, as the dIrr galaxies have more intense star formation or richer metallicity. There is a weak anticorrelation between the cold dust-to-stellar mass ratio and the specific star formation rate for our galaxies.
\end{abstract}

Unified Astronomy Thesaurus concepts: Dwarf irregular galaxies (417); Interstellar medium (847); Interstellar dust (836); Submillimeter astronomy (1647)

\section{Introduction}

Interstellar dust plays a key role in star formation and galaxy evolution. It absorbs the photons of stellar and ionized gas radiating in the ultraviolet (UV) and visible wavelengths then reemits them in infrared (IR) wavelengths (Rémy-Ruyer et al. 2013). Understanding how dust properties vary with galaxy global properties may provide important constraints on galaxy evolution. Most of the dust emission comes from the cold phase (Galametz et al. 2012) and cold dust properties can be inferred from the spectral energy distribution (SED) of galaxies in the IR to submillimeter wavelength ranges (Hermelo et al. 2013).

The commonly used SED model to fit the dust emission is the modified blackbody (MBB) model generally including the single temperature modified blackbody (SMBB) model and the two-temperature modified blackbody (TMBB) model, which is simple and flexible. The MBB model depends on the dust mass, temperature $T$ and emissivity index $\beta$. An anticorrelation between $T$ and $\beta$ has often been found (Dupac et al. 2003; Désert et al. 2008; Shetty et al. 2009a, 2009b; Galametz et al. 2012; Lamperti et al. 2019; Chang et al. 2020). This anticorrelation is likely artificial and is caused by noise in the data. Therefore, the estimated $T$ and $\beta$ are not very accurate leading to potential biases in the measured dust masses. In order to break the degeneracy between $T$ and $\beta$, the hierarchical
Bayesian approach is introduced to fit the MMB model (Kelly et al. 2012; Juvela et al. 2013; Veneziani et al. 2013; Galliano 2018; Lamperti et al. 2019), which allows us to study the independent relation of these two dust quantities with other global galaxy properties.

In nearby galaxies, cold dust properties vary with galaxies properties, for example metallicity, star formation rate (SFR), and stellar mass (Galametz et al. 2010, 2014; Cortese et al. 2014; Lamperti et al. 2020). An excess emission at submillimeter wavelengths is found in low-metallicity galaxies such as dwarf galaxies (Bot et al. 2010; Gordon et al. 2010; Galametz et al. 2011; Galliano et al. 2011). The submillimeter excess is defined as extra emission of the observation compared to the prediction derived from fitting the SED in the infrared/submillimeter range using a single $\lambda^{-\beta}$ emissivity law (Galametz et al. 2014). A $500 \mu \mathrm{m}$ excess is often detected in dwarf irregular (dIrr) galaxies (Dale et al. 2012; Rémy-Ruyer et al. 2013). Nevertheless, the submillimeter excess emission is sometimes probed apparently beyond Herschel bands stressing the necessity of observation longer than $500 \mu \mathrm{m}$ to properly investigate the presence of submillimeter excess (Rémy-Ruyer et al. 2013). What cause submillimeter excess is still an open debate by now. Some studies support the view that the submillimeter excess is associated with an additional dust component not included in current models, for example, a very cold dust component with temperature below 10 K (Chini et al. 1995; Krugel et al. 1998; 
Popescu et al. 2002), spinning dust emission or magnetic grains (Draine \& Lazarian 1998; de Oliveira-Costa et al. 2004; Finkbeiner et al. 2004; Casassus et al. 2006; Dobler \& Finkbeiner 2008; Miville-Deschênes et al. 2008), while other studies suggest that those components are not enough to cause the excess emission (Reach et al. 1995; Lisenfeld et al. 2002; Draine \& Hensley 2012). Some studies show that in some cases the submillimeter excess can be explained by non-dust components, such as $\mathrm{CO}(3-2)$ line, free-free or synchrotron emission (Galametz et al. 2014; Izotov et al. 2014), while other studies find that in some cases non-dust components are not enough to explain the submillimeter excess (Galliano et al. 2003; Chang et al. 2020). It is necessary to conduct more studies linking the submillimeter excess with cold dust properties, gas mass fraction, and fundamental galaxy properties to understand the origins of such excess or at least to know in which situation the excess will be easily detected. We investigate the potential submillimeter excess emission at 500 and $850 \mu \mathrm{m}$ in 12 nearby dIrr galaxies. For the submillimeter ${ }^{11}$ completeness, we also investigate the $350 \mu \mathrm{m}$ excess emission.

This paper is organized as follows. In Section 2, we present the sample and the data set we used. In Section 3, we present the global flux densities, the TMBB model, and the hierarchical Bayesian technique, performing SED fitting and results for our galaxies. In Section 4, we compare the 350, 500, and $850 \mu \mathrm{m}$ observed flux and modeled flux, investigate how the difference between the observation and extrapolation at $850 \mu \mathrm{m}$ correlates with gas mass fraction, cold dust parameters, and fundamental galaxy parameters of our galaxies and analyze the properties of cold dust parameters (temperature, dust emissivity index, and mass). In Section 5, we finally summarize the main conclusions of this study.

\section{Sample and Data}

\subsection{Sample}

In this paper, we study nearby irregular galaxies from the DustPedia project ${ }^{12}$ (Davies et al. 2017; Clark et al. 2018). First, we select those irregular galaxy that have photometry of at least six wavelengths from mid-infrared to submillimeter $(22,60 / 70,100,160,250,350$, and $500 \mu \mathrm{m})$ excluding the galaxies with a major flag associated with the flux densities due to their contamination, artefacts, or insufficient sky coverage (flags "C," "A," "N," respectively, given in the aperture photometry table from the DustPedia project). Second, we check the $850 \mu \mathrm{m}$ data of the selected irregular galaxies. For the galaxies that have known photometry at $850 \mu \mathrm{m}$ from the Second Planck Catalog of Compact Sources (Planck CCS2), ${ }^{13}$ those galaxies with signal-to-noise $\mathrm{S} / \mathrm{N}=$ Flux $/$ Flux $_{\text {err }} \geqslant 3$ are preserved. For the remaining galaxies which are not present in the Planck CCS2 catalog but have $850 \mu \mathrm{m}$ images, we measure their $850 \mu \mathrm{m}$ flux densities from the maps (see Section 3.1 for details) and retain the galaxies with $S / N \geqslant 3$. We thus get 13 nearby irregular galaxies with data ranging from $22-850 \mu \mathrm{m}$. Third, we check that the 13 galaxies have similar stellar mass and SFR to make sure they belong to the same population. This

\footnotetext{
11 The submillimeter range is defined as wavelengths from about $300-1000 \mu \mathrm{m}$ (Dale et al. 2012).

12 DustPedia is funded by the European Union, as a European Research Council (ERC) 7th Framework Program (FP7) project (PI: Jon Davies, proposal 606824): http://dustpedia.astro.noa.gr.

${ }^{13}$ https://irsa.ipac.caltech.edu/cgi-bin/Gator/nph-scan?submit=Select\& projshort $=$ Planck
}

is a requirement of the hierarchical Bayesian approach that we want to use for the SED fitting (see Lamperti et al. 2019 for details). After this check, we exclude one galaxy that has stellar mass and an SFR too low (by $>0.5 \mathrm{dex}$ ) compared to the rest of the sample. According to the latest definition of dwarf galaxies (Bullock \& Boylan-Kolchin 2017; Kong et al. 2019), we classify our irregular galaxies into bright dIrr galaxies, which is a type of dwarf galaxies with the largest stellar masses (Bullock \& Boylan-Kolchin 2017).

\subsection{Data}

Our data set consists of photometric points at $22 \mu \mathrm{m}$ (Widefield Infrared Survey Explorer (WISE)), $60 \mu \mathrm{m}$ (IRAS) $/ 70 \mu \mathrm{m}$ (Spitzer or Herschel/PACS), $100 \mu \mathrm{m}$ (IRAS or Herschel/PACS), $160 \mu \mathrm{m}$ (Herschel/PACS), 250, 350, $500 \mu \mathrm{m}$ (Herschel/Spectral and Photometric Imaging Receiver (SPIRE)), and $850 \mu \mathrm{m}$ (Planck). We also used auxiliary photometry at $12 \mu \mathrm{m}$ observed by WISE (see Appendix A). The details about the observations and data reduction can be found in Clark et al. (2018).

In this paper, in order to measure $850 \mu \mathrm{m}$ flux density, we use the data from Planck 2018 results (Planck Collaboration et al. 2020a) based on the continuous observation between 2009 August 12 and 2013 October 23, and download them from the Planck Legacy Archive. ${ }^{14}$ The maps at $850 \mu \mathrm{m}$ are provided in HEALPIX format (Górski et al. 2005) with resolution labeled by the $N_{\text {side }}$ value of 2048 corresponding to a pixel size of $1.71^{\prime}$. The FWHM of the effective beam of the Gaussian is 4.94' (see Table 12 in Planck Collaboration et al. 2020a). The dipole absolute calibration accuracy at $850 \mu \mathrm{m}$ is 0.024\% (listed in Table 12 in Planck Collaboration et al. 2020a). Their high frequency instrument data processing and calibration procedures are described in detail in Planck Collaboration et al. (2020a) and Planck Collaboration et al. (2020b). Based on our goal of extracting the flux density, we use the Stokes $I$ maps (Planck Collaboration et al. 2020a). Those image maps of our galaxies as cutout areas from sky map are in gnomonic reprojection. The unit of these maps is converted from cosmic microwave background (CMB) temperature (Kelvin) to jansky per steradian when they are downloaded from the Planck Legacy Archive. We also chose to subtract the CMB when downloading those maps. There are emission of other components (e.g., cirrus and cosmic infrared background) in the map (Hermelo et al. 2016). Because there is no matching data of those components released in Planck 2018 results, we cannot subtract them when downloading the map. According to Hermelo et al. (2016), we subtract them from every pixel of the image as the mean value of background in the annulus around the source (see Section 3.1).

\section{Global SED Fitting}

\subsection{Global Flux Densities at All Bands}

The global flux densities at 22-500 $\mu \mathrm{m}$ we used are obtained from Clark et al. (2018), which are extracted from aperturematched photometry (see their Section 3 for details). No color corrections are applied to these flux densities at this stage since the color corrections rely on the shape of the SED. Thus, the color corrections are applied as part of the SED fitting procedure (see the description in Section 3.4). We ignored the line contamination such as [N II] $205 \mu \mathrm{m}$ or CO at SPIRE bands and free-free + synchrotron emission at wavelengths

\footnotetext{
${ }^{14}$ http://pla.esac.esa.int/pla/\#maps
} 
shortward of $850 \mu \mathrm{m}$ due to their minor contribution compared to continuum emission (Galametz et al. 2014; Hermelo et al. 2016; Chang et al. 2020).

In our sample, the $850 \mu \mathrm{m}$ flux densities of only three galaxies (IC 0010, NGC 4214 and NGC 4449) found in Planck CCS2 are detected with $\mathrm{S} / \mathrm{N} \geqslant 3$ (see Section 2.1). For the remaining galaxies, we measured the $850 \mu \mathrm{m}$ flux density according to the method of aperture photometry described at length in Clark et al. (2018) and Planck Collaboration et al. (2014), using a circular aperture centering the position of source with radius equal to its average FWHM of the effective beam (described in Section 2.2) and annulus around this circular aperture for background with an inner radius of 1 time FWHM and an outer radius of 2 times FWHM. The annulus of background is used to evaluate the level of the background and make a local estimate of the noise to calculate the uncertainty in the estimate of the flux density. We extract the $850 \mu \mathrm{m}$ flux density using the following formula (Planck Collaboration et al. 2014):

$$
S_{\mathrm{obs}}=\left(S_{\mathrm{ap}}-S_{\mathrm{an}}\left(\frac{k_{0}^{2}}{k_{2}^{2}-k_{1}^{2}}\right)\right),
$$

where $k_{o}$ is the radius of the circular aperture, $k_{1}$ and $k_{2}$ are the inner and outer radius of the annulus, and $S_{\text {ap }}$ and $S_{\text {an }}$ are the flux densities of the galaxy in the circular aperture and annulus. The flux densities $\left(S_{\text {obs }}\right)$ need to be corrected due to the both flux densities of the source missing from the circular aperture and that eliminated through background subtraction (Planck Collaboration et al. 2014). We use the formula below to correct the $S_{\text {obs }}$ to the $S_{\text {true }}$ (Planck Collaboration et al. 2014):

$$
S_{\mathrm{obs}}=\left(\frac{4 \Omega_{\mathrm{FWHM} 1}-\Omega_{\mathrm{FWHM} 2}}{3 \Omega}\right) S_{\text {true }},
$$

where $\Omega$ is the beam solid angle $\left(27.69 \operatorname{arcmin}^{2}\right.$, see Planck Collaboration et al. 2016b), and $\Omega_{\mathrm{FWHM} 1}\left(25.32 \mathrm{arcmin}^{2}\right.$, see Planck Collaboration et al. 2016b) and $\Omega_{\text {FWHM2 }}$ (27.53 $\mathrm{arcmin}^{2}$, see Planck Collaboration et al. 2016b) are the solid angles of the beam within the radius equal to 1 and 2 times the effective FWHM, respectively.

Following Hermelo et al. (2016), we consider the calibration uncertainties, $\Delta_{\mathrm{cal}, \nu} / f_{\nu} \sim 0.024 \%$ at $850 \mu \mathrm{m}$ (see Section 2.2) and measurement uncertainty resulted from background fluctuations, $\Delta_{\text {back}}$, calculated from $\Delta_{\text {back }}=\sigma_{\text {back }}$ $\sqrt{N_{\text {aper }}+N_{\text {aper }}^{2} / N_{\text {back }}}$ (detailed in Section 3.4 of Hermelo et al. 2016) with $\sigma_{\text {back }}$ the standard deviation of background noise, $N_{\text {aper }}$ the pixel number in source aperture, and $N_{\text {back }}$ the pixel number in the annulus for background. The quadratic sum of $\Delta_{\text {cal }}$ and $\Delta_{\text {back }}$ are calculated as final uncertainty of flux density, $\Delta_{\text {final }}=\sqrt{\Delta_{\text {cal }}^{2}+\Delta_{\text {back }}^{2}}$. The measured $850 \mu \mathrm{m}$ flux densities with their uncertainties are listed in Table 2 in Appendix A.

It is significant to note that the $850 \mu \mathrm{m}$ emission consists of three components (Hermelo et al. 2016): (1) dust emission, (2) free-free emission and synchrotron emission, (3) $\mathrm{CO}(3-2)$ line emission, which falls into the Planck $850 \mu \mathrm{m}$ filter. In order to probe the dust emission excess at $850 \mu \mathrm{m}$, those contributions of non-dust components need to be removed. The non-dusty emission correction for $850 \mu \mathrm{m}$ flux densities is shown in Appendix A. The fluxes at $850 \mu \mathrm{m}$ corrected for $\mathrm{CO}(3-2)$ line, synchrotron and free-free emission with their uncertainties are listed in the Table 5 in Appendix B.

\subsection{The TMBB Model}

The SMBB model usually overestimates cold dust temperatures due to the non-negligible contribution of the warm dust at 24-70 $\mu \mathrm{m}$ (Galametz et al. 2012). To constrain the warm dust emission, we apply a TMBB model (Galametz et al. 2012; Lamperti et al. 2019; Chang et al. 2020). The model assumes that dust grains are optically thin. In fact, dust models show that this assumption is suitable for the wavelengths $\geqslant 100 \mu \mathrm{m}$, while the dust grains may be optically thick at shorter wavelengths (Draine \& Li 2007). However, some studies suggest that even if the dust is optically thick, on global galaxy scales, it has no much effect on the SED shape at $22 \mu \mathrm{m}$ (Lamperti et al. 2019 and references therein). The TMBB model is defined as follows (Lamperti et al. 2019):

$$
F_{\lambda}=\frac{M_{\mathrm{w}}}{D^{2}} \kappa_{0}\left(\frac{\lambda_{0}}{\lambda}\right)^{\beta_{\mathrm{w}}} B_{\nu}\left(\lambda, T_{\mathrm{w}}\right)+\frac{M_{\mathrm{c}}}{D^{2}} \kappa_{0}\left(\frac{\lambda_{0}}{\lambda}\right)^{\beta_{\mathrm{c}}} B_{\nu}\left(\lambda, T_{\mathrm{c}}\right)
$$

each component being modeled as one MBB model, where $B_{\nu}$ is the Planck function, $M_{\mathrm{w}}$ and $M_{\mathrm{c}}$ are the warm and cold dust mass, $D$ is the distance of the galaxy, $\beta_{\mathrm{w}}$ and $\beta_{\mathrm{c}}$ are the emissivity index of the warm and cold component, $T_{\mathrm{w}}$ and $T_{\mathrm{c}}$ are the temperature of the warm and cold component, and $\kappa_{0}$ is the reference dust mass absorption coefficient at $\lambda_{0}$. The $\kappa_{0}$ is taken as a constant value $\kappa_{0}=\kappa(500 \mu \mathrm{m})=0.051 \mathrm{~m}^{2} \mathrm{~kg}^{-1}$ (Clark et al. 2016). In order to reduce the number of free parameters in our model, we fix $\beta_{\mathrm{w}}$ to 2 . We note that the $\beta_{\mathrm{w}}$ assumption has a negligible effect on the cold dust temperature for our galaxies. We tested that assuming $\beta_{\mathrm{w}}=1$ (or $\beta_{\mathrm{w}}=1.5$ ) instead of $\beta_{\mathrm{w}}=2$, the cold dust temperatures decrease by less than $1.1 \%$ on average, and these variations are smaller than the uncertainties on $T_{\mathrm{c}}$. This result is similar to that of Galametz et al. (2012), which shows that their choice of $\beta_{\mathrm{w}}$ does not effect the $T_{\mathrm{c}}$ and also adopt $\beta_{\mathrm{w}}=2$ in a similar TMBB model. For the emissivity index of the cold component $\left(\beta_{\mathrm{c}}\right)$, we adopt $\beta_{\mathrm{c}}=2$, which allows us to compare our results with other works, to define and identify potential submillimeter excess at 350-850 $\mu \mathrm{m}$. The value of $\beta_{\mathrm{c}}=2$ is used here mainly because that has been the standard value used in the previous studies and it is consistent with the results derived from standard dust model (Desert et al. 1990; Li \& Draine 2001; Relaño et al. 2018). The TMBB model with $\beta_{\mathrm{c}}=2$ can account for the thermal emission modeled using dust grains with standard properties (Galametz et al. 2014) and can model the SEDs of nearby galaxies reasonably well (Dunne $\&$ Eales 2001; Klaas et al. 2001). Generally, $\beta=2$ in MBB models is assumed for most works done previously to study submillimeter excess (Rémy-Ruyer et al. 2013; Galametz et al. 2014; Chang et al. 2020). Referring to Galametz et al. (2014), we also use the free $\beta_{\mathrm{c}}$ assumption for the purpose of testing which values could explain the submillimeter slopes and detect potential strong flattening of the submillimeter slope in our galaxies. In this model, there are five free parameters $\left(\beta_{\mathrm{c}}, M_{\mathrm{c}}, T_{\mathrm{c}}, M_{\mathrm{w}}\right.$, and $\left.T_{\mathrm{w}}\right)$. The cold component of the TMBB model describes the emission of the cold dust as an MBB model with a single temperature. The $T_{\mathrm{c}}$ and $M_{\mathrm{c}}$ here should be thought as an approximation of the actual cold temperature and mass structure of our galaxies. The $\beta_{\mathrm{c}}$ here is not an intrinsic emissivity index of one grain population but the sum of different grain populations, which is more accurately the effective emissivity index and is identical to the 
intrinsic emissivity indices in the isothermal case. Details can be found in Galametz et al. (2012).

\subsection{The Hierarchical Bayesian Technique}

Recently, Lamperti et al. (2019) used a hierarchical Bayesian approach to reduce the $T-\beta$ degeneracy effectively to obtain more accurate measurements of the dust parameters. Using the hierarchical Bayesian method, we can express the posterior probability function of the dust parameters for the $n$ galaxies in a sample as (Lamperti et al. 2019):

$$
\begin{aligned}
p & \left(\boldsymbol{\theta}_{1}, \ldots, \boldsymbol{\theta}_{n}, \boldsymbol{\mu}, \Sigma \mid \boldsymbol{F}_{1}^{\mathrm{obs}}, \ldots, \boldsymbol{F}_{n}^{\mathrm{obs}}\right) \\
& \propto \prod_{i=1}^{n} p\left(\boldsymbol{\theta}_{i} \mid \boldsymbol{F}_{i}^{\mathrm{obs}}, \boldsymbol{\mu}, \Sigma\right) \cdot p(\boldsymbol{\mu}) \cdot p(\Sigma) \\
& \propto \prod_{i=1}^{n} p\left(\boldsymbol{F}_{i}^{\mathrm{obs}} \mid \boldsymbol{\theta}_{i}\right) \cdot p\left(\boldsymbol{\theta}_{i} \mid \boldsymbol{\mu}, \Sigma\right) \cdot p(\boldsymbol{\mu}) \cdot p(\Sigma),
\end{aligned}
$$

where $\boldsymbol{F}^{\mathrm{obs}}$ is the vector containing the flux emission at each wave band. $\boldsymbol{\mu}$ and $\Sigma$ are called hyperparameters and describe the prior distribution of the parameters $\boldsymbol{\theta} . \boldsymbol{\mu}$ is the average of the parameter vector $\boldsymbol{\theta}$ and $\Sigma$ is the covariance matrix describing the standard deviation and correlation of $\boldsymbol{\theta} \cdot p(\boldsymbol{\mu})$ and $p(\Sigma)$ are the prior distributions of the hyperparameters. In order to define the prior distributions of the parameters given the hyperparameters, a multivariate Student's t-distribution with $f=8$ degrees of freedom is adopted (see Lamperti et al. 2019 for details):

$$
p\left(\boldsymbol{\theta}_{i} \mid \boldsymbol{\mu}, \Sigma\right)=\operatorname{MultiStudent}\left(\boldsymbol{\theta}_{i} \mid \boldsymbol{\mu}, \Sigma, f=8\right) .
$$

For the noise distribution, we use a normal distribution (see Lamperti et al. 2019 for details). We assume that the monochromatic likelihood of every galaxy $i$ follows a normal distribution. The form of the normal distribution for each wave band $j=1, \cdots, m$ is (Lamperti et al. 2019)

$$
p\left(F_{i, j}^{\mathrm{obs}} \mid \boldsymbol{\theta}_{i}, F_{i, j}^{\mathrm{err}}, \delta_{j}\right)=\operatorname{normal}\left(F_{i, j}^{\mathrm{obs}} \mid F_{i, j}^{\bmod }\left(\boldsymbol{\theta}_{i}\right), F_{i, j}^{\mathrm{err}}\right)
$$

where $\delta_{j}$ is the calibration offset in the $j$ th wave band. $F_{i, j}^{\bmod }\left(\boldsymbol{\theta}_{i}\right)$ is the modeled flux assuming the TMBB model as described in Equation (3).

\subsection{Performing the SED Fittings and the Results}

To apply the hierarchical Bayesian method to the SED fitting, we use $\operatorname{Stan}^{15}$ (Carpenter et al. 2017), which is a software for Bayesian inference employing the No-U-Turn sampler and runs PyStan ${ }^{16}$, which is the Python interface to Stan (Stan Development Team 2018). A detailed description can be found in Lamperti et al. (2019). Beam corrections and color corrections (also called filter corrections) have been applied to the Herschel fluxes using an iterative approach following Lamperti et al. (2019). These corrections depend on the shape of the SED, which is described by the values of temperature and $\beta$. For each galaxy, we first ran the SED fitting to derive $T$ and $\beta$, then we applied the beam and color corrections using the derived $T$ and $\beta$ values. Then rerun the SED fitting using the corrected fluxes. The details can be found in Lamperti et al. (2019). These corrections are small when compared with uncertainties of the fluxes, thus they do not significantly affect our results.

Our aim is to explore potential submillimeter excess at 350 , 500 , and $850 \mu \mathrm{m}$, so we carry out three sets of SED fits using

\footnotetext{
15 http://mc-stan.org/

16 http://pystan.readthedocs.io/en/latest/
}

data in the $22-250 \mu \mathrm{m}$ range, $22-350 \mu \mathrm{m}$ range and 22-500 $\mu \mathrm{m}$ range, respectively, referring to Galametz et al. (2014) and Chang et al. (2020). The fitting parameters $\left(\beta_{\mathrm{c}}, M_{\mathrm{c}}\right.$, $T_{\mathrm{c}}, M_{\mathrm{w}}$, and $T_{\mathrm{w}}$ ) and their corresponding errors can be inferred from their respective posterior distributions. The median values of the marginalized posterior probability distributions are taken as the result for parameters and the uncertainties are estimated from the values corresponding to the 16th and 84th percentiles. The model fluxes are calculated using the fitting result of parameters above by Equation (3). To estimate the uncertainties on the SED modeled fluxes, we consider the parameters with likelihood values in the highest 68th percentile according to Lamperti et al. (2019). Then we estimate the SED modeled fluxes using these parameters and we use the minimum and maximum flux values to estimate the uncertainty range. The modeled fluxes we derived at 350,500 , and $850 \mu \mathrm{m}$ and their uncertainties in the case of $\beta_{\mathrm{c}}=2$ or free for each galaxy are listed in Tables 3-5 in Appendix B, respectively.

Figure 1 shows an example of the hierarchical Bayesian SED fitting for IC 3268 using the TMBB model in the case of $\beta_{\mathrm{c}}=2$ (the first line panels) and $\beta_{\mathrm{c}}$ free (the second line panels). The SEDs of the remaining 11 galaxies are shown in Figure 10 in Appendix D. The SED fittings, which were done using data sets of $22-250 \mu \mathrm{m}, 22-350 \mu \mathrm{m}$ and $22-500 \mu \mathrm{m}$ to predict the flux at 350,500 , and $850 \mu \mathrm{m}$, respectively, are shown in the panels from left to right. The shadow area represents the lower and upper uncertainties of the modeled flux. Obviously, using $\beta_{\mathrm{c}}$ as free, the uncertainty of the modeled flux density is larger than that using $\beta_{\mathrm{c}}$ fixed to 2 (see the shadow). For this galaxy, the models could match the observation within uncertainty at 350 and $500 \mu \mathrm{m}$. There is an excess emission at $850 \mu \mathrm{m}$ in both cases of $\beta_{\mathrm{c}}$ (see the red point). We will discuss the excess at submillimeter in detail in the next section.

\section{Analysis and Discussion}

\subsection{Comparing Prediction with Observation}

Following Chang et al. (2020), we define the submillimeter excess as the observed flux above the modeled flux based on the TMBB model of $\beta_{\mathrm{w}}=2$ and $\beta_{\mathrm{c}}=2$ (see Section 3.2 for details) in the same band taking into account uncertainties of both observations and predictions. Additionally, we will also compare the observed flux with the modeled flux derived from the TMBB model with a free $\beta_{\mathrm{c}}$. Specifically, we consider a galaxy to have a submillimeter excess in a particular band when the following condition is satisfied for that band:

$$
F^{\text {obs }}-F^{\text {model }}>F_{\text {err }}^{\text {obs }}+F_{\text {err }}^{\text {model }} .
$$

In other words, if the residual between observation and prediction at 350,500 , and $850 \mu \mathrm{m}$ is greater than the sum of both error ( $1 \sigma$ criteria), there is an excess at those submillimeter bands (Rémy-Ruyer et al. 2013, 2015).

We find that the TMBB model with $\beta_{\mathrm{c}}=2$ matches the observations within uncertainties at $350 \mu \mathrm{m}$ for all galaxies. Therefore, the observed $350 \mu \mathrm{m}$ emission in our galaxies can be accounted for by thermal emission modeled using dust grains with standard properties. At $500 \mu \mathrm{m}$, only NGC 4214 shows an excess emission based on $\beta_{\mathrm{c}}=2$. However, using a flatter $\beta_{\mathrm{c}}$ $\left(\beta_{\mathrm{c}}<2.0\right)$ can provide a prediction matching the observed flux within uncertainty. This may suggest that different properties of the dust grains or a different dust composition in this galaxy can explain the excess emission. There are nine galaxies (IC 3268, 

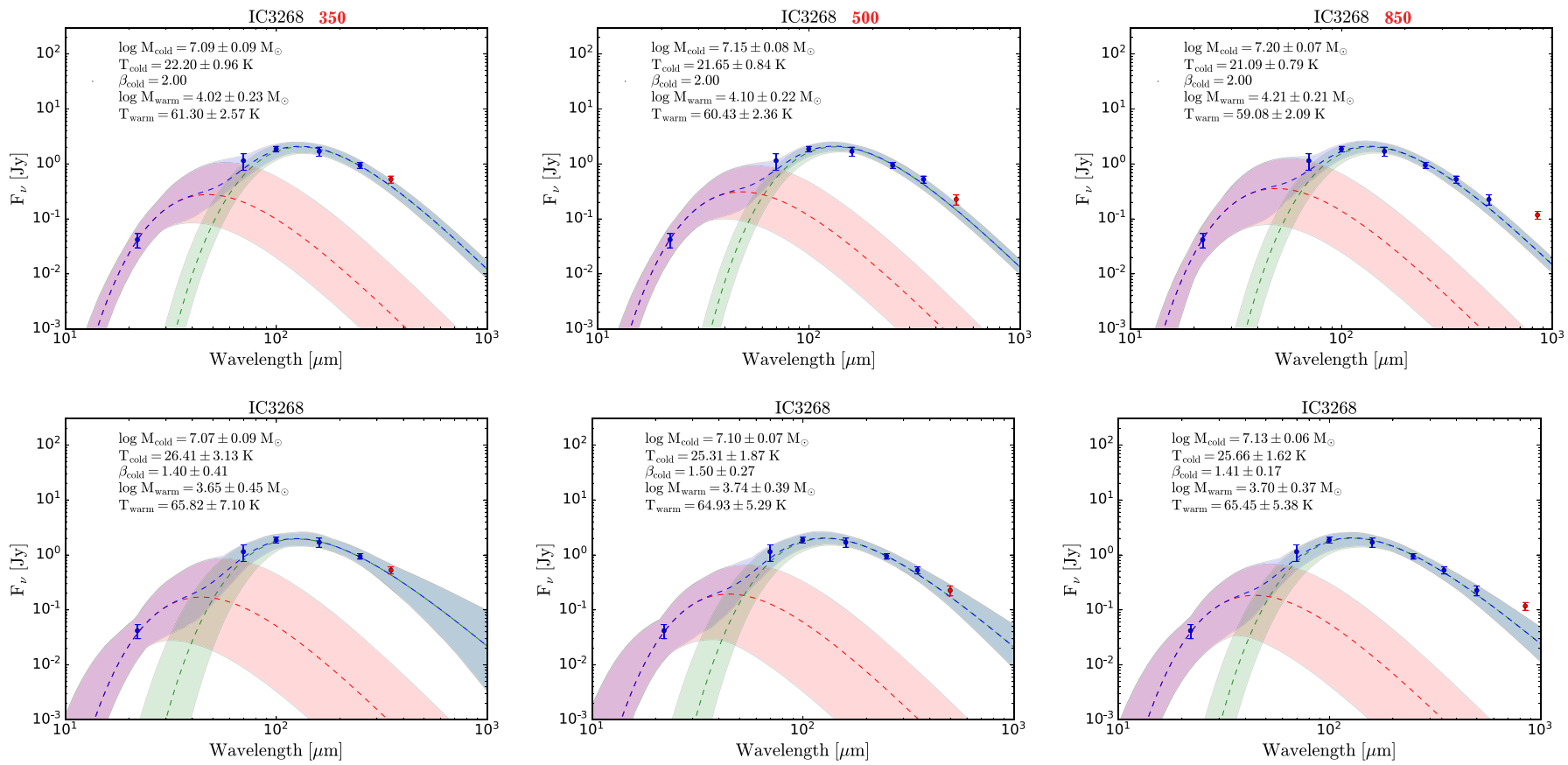

Figure 1. An example of global SEDs of IC 3268 obtained using the TMBB model ( $\beta_{\mathrm{w}}=2$ and $\beta_{\mathrm{c}}=2$ or $\beta_{\mathrm{c}}$ as free) with the hierarchical Bayesian technique. The first line panels show the SED fits in the case of $\beta_{\mathrm{c}}=2$ and the second line panels show the SED fits in the case of $\beta_{\mathrm{c}}$ as free, from left to right, using the data ranging from $22-250 \mu \mathrm{m}$ to predict $350 \mu \mathrm{m}$ flux, from $22-350 \mu \mathrm{m}$ to predict $500 \mu \mathrm{m}$ flux and from 22-500 $\mu \mathrm{m}$ to predict $850 \mu \mathrm{m}$ flux, respectively. The fitting result of the parameters is shown in the upper left corner of each panel. The warm component (with $\beta_{\mathrm{w}}$ fixed to 2) is overlaid in red and the cold component (with $\beta_{\mathrm{c}}$ fixed to 2 or free) is in green. The blue area represents the sum of warm component and cold component. The dotted line represents the model flux. The shaded regions show the lower and upper $1 \sigma$ uncertainties on the SED models. WISE, Herschel, or Planck data (included in the fitting procedures) are overlaid with blue solid circles. The red solid circles (not included in the fitting) represent the observations at 350,500, or $850 \mu \mathrm{m}$ shown in the panel from left to right, where the observed Planck flux density at $850 \mu \mathrm{m}$ has been corrected for $\mathrm{CO}(3-2)$ line, free-free and synchrotron emission.

IC 3476, NGC 1427A, NGC 4032, NGC 4214, NGC 4376, NGC 4449, NGC 4630, and NGC 7694) showing excess emission at $850 \mu \mathrm{m}$ based on $\beta_{\mathrm{c}}=2$. It is interesting that five of them (IC 3268, NGC 1427A, NGC 4032, NGC 4376, and NGC 7694) still exhibit a clear $850 \mu \mathrm{m}$ excess when $\beta_{\mathrm{c}}$ is free.

Before comparing the observations at $850 \mu \mathrm{m}$ with extrapolations, the contributions of $\mathrm{CO}(3-2)$ line, synchrotron and free-free emission to the total flux at $850 \mu \mathrm{m}$ have been removed except for NGC 1427A. The total $850 \mu \mathrm{m}$ flux density of NGC 1427A has been only corrected for the $\mathrm{CO}(3-2)$ line emission since the mid-frequency radio flux were not found to extrapolate the free-free and synchrotron emission at $850 \mu \mathrm{m}$ (see Appendix A). The excess emission at $850 \mu \mathrm{m}$ are not accounted for by those non-dust contributions for our galaxies. However, for NGC 1427A, we cannot determine whether synchrotron or free-free emission is the cause of excess emission at $850 \mu \mathrm{m}$.

\subsection{The Correlation between $850 \mu m$ Excess and Physical Parameters}

In this section, we investigate how the differences between the $850 \mu \mathrm{m}$ observed and modeled flux densities vary with gas mass fraction, cold dust parameters, and fundamental galaxy properties of our galaxies, to identify under which situation the $850 \mu \mathrm{m}$ excess is prominent. This will shed light onto the circumstances that might be more likely to make galaxy show submillimeter excess at $850 \mu \mathrm{m}$. Following Galametz et al. (2014) and Chang et al. (2020), we characterize the differences between the $850 \mu \mathrm{m}$ observed and modeled flux densities as the ratios between them, which are listed in Table 5 in Appendix B. Those $850 \mu \mathrm{m}$ observed flux densities here have been corrected for the non-dust emission. We note that for one galaxy (NGC 1427A), we could not subtract the free-free and synchrotron emission at $850 \mu \mathrm{m}$ because of the lack of mid-frequency radio data (See Appendix A for a detailed explanation). Since only one galaxy shows an excess emission at $500 \mu \mathrm{m}$, and no galaxy shows excess emission at $350 \mu \mathrm{m}$, we only analyze $850 \mu \mathrm{m}$ emission in this work (see Sections 4.2.1-4.2.3). It should be noted that our following discussions are based on the limited number of galaxies in our sample.

\subsubsection{The Correlation between $850 \mu \mathrm{m}$ Excess and Gas Mass Fraction}

The gas mass fraction is defined as the mass of hydrogen gas divided by the stellar mass (molecular hydrogen gas mass fraction $M_{\mathrm{H}_{2}} / M_{*}$ and atomical hydrogen gas mass fraction $\left.M_{\mathrm{H} \mathrm{I}} / M_{*}\right)$. In Figure 2, we plot the $850 \mu \mathrm{m}$ observed-tomodeled flux density ratios in the case of $\beta_{\mathrm{c}}=2$ or free as functions of $M_{\mathrm{H}_{2}} / M_{*}$ and $M_{\mathrm{H} \mathrm{I}} / M_{*}$. These parameters are listed in Table 1. There is an anticorrelation between $850 \mu \mathrm{m}$ observed-to-modeled ratios and $M_{\mathrm{H}_{2}} / M_{*}$ in the case of $\beta_{\mathrm{c}}=2$ with a Pearson correlation coefficient ${ }^{17} R_{\text {pear }}=-0.42$ and weak anticorrelation in the case of free $\beta_{\mathrm{c}}$ with $R_{\text {pear }}=-0.34$ for our galaxies (see the top panel in Figure 2). Moreover, for

\footnotetext{
17 The Pearson correlation coefficient, $R_{\text {pear }}$, indicates the degree of linear correlation between two variables. The larger the absolute value of $R_{\text {pear }}$ is, the stronger the correlation is. The classification of absolute value of $R_{\text {pear }}: 0.0-0.2$ no correlation, $0.2-0.4$ weak correlation, $0.4-0.6$ medium correlation, $0.6-0.8$ strong correlation, and $0.8-1.0$ highly strong correlation. If $R_{\text {pear }}$ is larger than zero, it means that the two variables are positively correlated. if $R_{\text {pear }}$ is smaller than zero, it means that the two variables are negatively correlated.
} 
Table 1

Sample Information

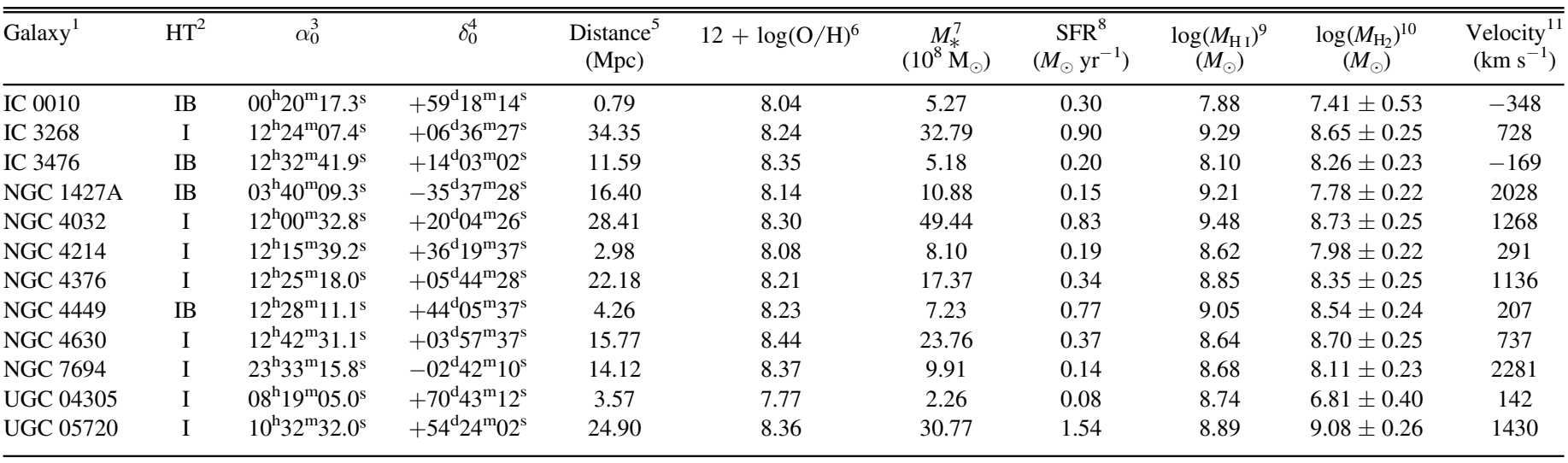

Note. (1) Galaxy name, (2) morphological classification of Hubble type (HT), (3) J2000.0 Right ascension, (4) J2000.0 decl., (5) the best distance, and (6) global oxygen abundance using PG16S calibration derived from DustPedia project, except for NGC 7694 and UGC 05720; the oxygen abundance of these two galaxies are calculated using the 10,143 star-forming regions detailed in De Vis et al. (2019) and Casasola et al. (2020), (7) the stellar mass, (8) the SFR, (9) the atomical hydrogen gas mass $\left(\mathrm{H}_{\mathrm{I}}\right)$ in $\log \left(M_{\odot}\right),(10)$ the molecular hydrogen gas mass $\left(\mathrm{H}_{2}\right)$ in $\log \left(M_{\odot}\right)$ calculated based on flux at $12 \mu$ m (see Appendix A), (11) velocity in kilometers per second derived from $\mathrm{NED}^{18}$. The values of Columns 2, 5, 7, 8, and 9 are derived from DustPedia project.

the galaxies showing $850 \mu \mathrm{m}$ excess emission, the $850 \mu \mathrm{m}$ observed-to-modeled flux density ratios and $M_{\mathrm{H}_{2}} / M_{*}$ show a stronger decreasing trend in the case of $\beta_{\mathrm{c}}=2$ with $R_{\text {pear }}=-0.77$ and a weak decreasing trend in the case of free $\beta_{\mathrm{c}}$ with $R_{\text {pear }}=-0.34$ (see the black and red triangles in the top panel). This may suggest that the excess emission at $850 \mu \mathrm{m}$ is easily detected in the dIrr galaxies with low molecular hydrogen gas mass fraction, i.e., the smaller $M_{\mathrm{H}_{2}} / M_{*}$ is, the stronger the $850 \mu \mathrm{m}$ excess is.

We find that the $850 \mu \mathrm{m}$ observed-to-modeled flux density ratio correlates with $M_{\mathrm{H}} / M_{*}$ for our sample in the case of $\beta_{\mathrm{c}}=2\left(R_{\text {pear }}=0.42\right)$ and in the case of free $\beta_{\mathrm{c}}\left(R_{\text {pear }}=0.37\right)$ in the bottom panel of Figure 2. Since $M_{\mathrm{HI}} / M_{*}$ is a proxy for how far the galaxies are progressing in their evolution (De Vis et al. 2017), this correlation suggests that the $850 \mu \mathrm{m}$ excess may depend on the evolutionary state of a galaxy. As galaxies evolve, the $M_{\mathrm{HI}} / M_{*}$ decreases and the $850 \mu \mathrm{m}$ excess emission tend to decrease or even disappear.

\subsubsection{The Correlation between $850 \mu m$ Excess and Cold Dust Parameters}

To study how the difference between the observation and extrapolation at $850 \mu \mathrm{m}$ varies with the cold dust parameters of our galaxies, we plot the ratios of $850 \mu \mathrm{m}$ observed-to-modeled flux densities in the case of $\beta_{\mathrm{c}}=2$ or free as functions of $M_{\mathrm{c}}$ and $T_{\mathrm{c}}$ in the left and middle panels of Figure 3 for our sample. We adopt the $M_{\mathrm{c}}$ and $T_{\mathrm{c}}$ derived from SED fitting with data ranging from $22-500 \mu \mathrm{m}$ since extrapolation at $850 \mu \mathrm{m}$ is derived using the results of the fit in this wavelength range. Those $T_{\mathrm{c}}$ and $M_{\mathrm{c}}$ are listed in Table 6 in Appendix C. The $850 \mu \mathrm{m}$ observation-to-prediction ratios do not show a particular trend with $M_{\mathrm{c}}$ in both $\beta_{\mathrm{c}}$ fixed to $2\left(R_{\text {pear }}=0.08\right)$ and free $\left(R_{\text {pear }}=0.20\right)$ in the left panel of Figure 3 . This suggests that the $850 \mu \mathrm{m}$ excess does not depend on the cold dust mass for our galaxies. There is no relation between the $850 \mu \mathrm{m}$ observation-to-prediction ratios and $T_{\mathrm{c}}$ in the case of $\beta_{\mathrm{c}}=2\left(R_{\text {pear }}=-0.19\right)$. A weak anticorrelation between those

\footnotetext{
18 http://ned.ipac.caltech.edu/forms/byname.html
}
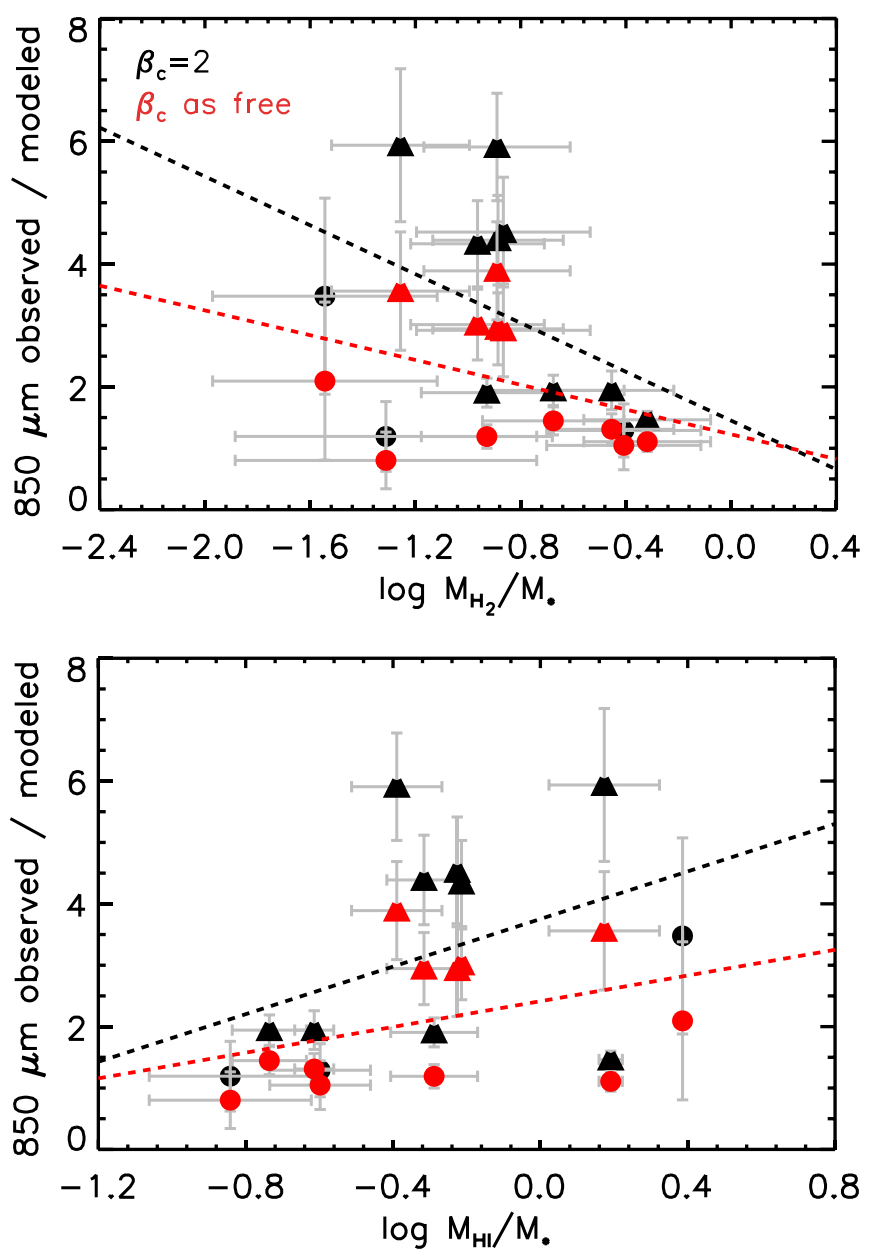

Figure 2. The correlations between the ratios of $850 \mu \mathrm{m}$ observed-to-modeled flux and molecular hydrogen gas mass fraction $M_{\mathrm{H}_{2}} / M_{*}$ (top) and atomical hydrogen gas mass fraction $M_{\mathrm{H}} / M_{*}$ (bottom) in the case of $\beta_{\mathrm{c}}=2$ (the black symbols) and $\beta_{\mathrm{c}}$ as free (the red symbols). The triangles represent the galaxies with $850 \mu \mathrm{m}$ excess. The circles show the galaxies without $850 \mu \mathrm{m}$ excess. The black and red lines fitted represent the trend. The gray lines represent the error bars. 

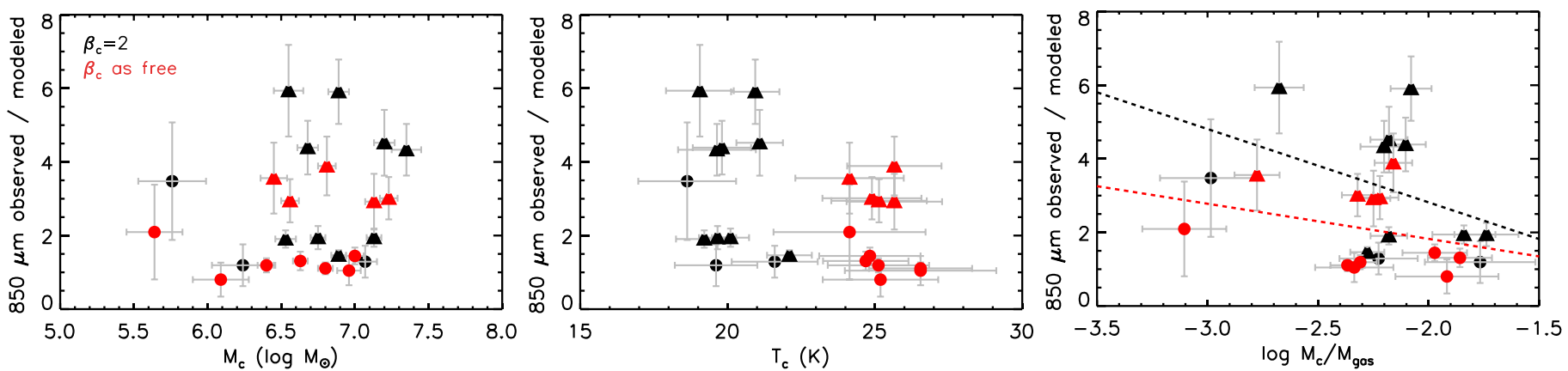

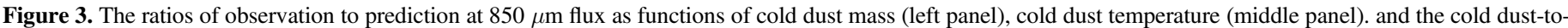
gas mass ratios (right panel) in the case of $\beta_{\mathrm{c}}=2$ or free. For the convention on colors and symbols, see Figure 2.

ratios and $T_{\mathrm{c}}$ is found in the case of $\beta_{\mathrm{c}}$ as free $\left(R_{\mathrm{pear}}=-0.28\right)$. However, we do not think that this anticorrelation is significant because the cold dust temperature values span a very small range (only $3 \mathrm{~K}$ ) when $\beta_{\mathrm{c}}$ is free. This indicates that the $850 \mu \mathrm{m}$ excess emission does not correlate with the cold dust temperature in our sample.

We also plot the ratios of $850 \mu \mathrm{m}$ observed-to-modeled flux densities in the case of $\beta_{\mathrm{c}}=2$ or free as a function of the ratio between cold dust mass and gas mass $\left(M_{\mathrm{c}} / M_{\text {gas }}\right.$, see the right panel of Figure 3). The dust-to-gas mass ratios play a key role in studying the circulation of substance in a galaxy. Since most of the dust in galaxies is in the cold phase, we can use the cold dust mass as the total dust mass directly (Galametz et al. 2012). Specifically, the fraction of warm dust mass to cold dust mass in our galaxies ranges from $0.05 \%-0.3 \%$ in the case of $\beta_{\mathrm{c}}=2$ and from $0.02 \%-0.6 \%$ in the case of free $\beta_{\mathrm{c}}$ in the range of $22-500 \mu \mathrm{m}$ SEDs. We sum the atomic and molecular hydrogen gas masses to obtain the total gas mass ( $\mathrm{H}$ I mass and $\mathrm{H}_{2}$ mass, see Galametz et al. 2011), which are listed in Table 1. As the $M_{\mathrm{c}} / M_{\text {gas }}$ decreases, the ratio of $850 \mu \mathrm{m}$ observed-to-modeled flux density tends to increase in the case of $\beta_{\mathrm{c}}=2\left(R_{\text {pear }}=-\right.$ $0.40)$ and free $\beta_{\mathrm{c}}\left(R_{\text {pear }}=-0.30\right)$ in our sample. Moreover, for the galaxies with $850 \mu \mathrm{m}$ excess, there is also an increasing trend of those ratios with decreasing $M_{\mathrm{c}} / M_{\mathrm{gas}}$ in the case of $\beta_{\mathrm{c}}=2\left(R_{\text {pear }}=-0.51\right)$. In the case of free $\beta_{\mathrm{c}}$, although there is no trend of those ratios with $M_{\mathrm{c}} / M_{\mathrm{gas}}$, the galaxies with $850 \mu \mathrm{m}$ excess have relatively low $M_{\mathrm{c}} / M_{\text {gas }}$ and high $850 \mu \mathrm{m}$ observed-to-modeled flux densities ratios in our galaxies (see the red triangles in the right panel of Figure 3). Our results suggest that the $850 \mu \mathrm{m}$ excess emission is more likely to occur in the dIrr galaxies with low $M_{\mathrm{c}} / M_{\text {gas }}$. The lower the $M_{\mathrm{c}} / M_{\mathrm{gas}}$ is, the stronger the $850 \mu \mathrm{m}$ excess is.

\subsubsection{The Correlation between $850 \mu \mathrm{m}$ Excess and the Fundamental Galaxy Properties}

To test how the difference between the observation and extrapolation at $850 \mu \mathrm{m}$ varies with the fundamental properties of galaxies, in Figure 4 we plot the ratios of observed to modeled at $850 \mu \mathrm{m}$ flux densities in the case of $\beta_{\mathrm{c}}=2$ or free as functions of (from left to right): the oxygen abundance, the stellar mass $\left(M_{*}\right)$ and specific star formation rate (sSFR). The $850 \mu \mathrm{m}$ observation-to-prediction ratios do not show a particular trend with the oxygen abundance in the case of $\beta_{\mathrm{c}}=2\left(R_{\text {pear }}=-0.07\right)$ or free $\left(R_{\text {pear }}=-0.02\right)$. A rather weak correlation between the $850 \mu \mathrm{m}$ observation-to-prediction ratios and $M_{*}$ is found in the case of $\beta_{\mathrm{c}}=2\left(R_{\text {pear }}=0.24\right)$ or free $\left(R_{\text {pear }}=0.33\right)$ for our galaxies. Since such correlation is very weak, it is not safe to say that $850 \mu \mathrm{m}$ excess is likely to be detected in our galaxies with higher $M_{*}$. Maybe the $850 \mu \mathrm{m}$ excess has no particular dependence on $M_{*}$.

There are strong anticorrelations between the $850 \mu \mathrm{m}$ observedto-modeled flux ratios and the sSFR in our sample in the case of $\beta_{\mathrm{c}}=2$ with $R_{\text {pear }}=-0.68$ and in the case of free $\beta_{\mathrm{c}}$ with $R_{\text {pear }}=-0.69$ (see the right panel of Figure 4) agreeing with the result of Relaño et al. (2018), which shows anticorrelation between their $500 \mu \mathrm{m}$ excess and SFR at spatial scales for the spiral galaxy $M_{33}$. If we consider only our dIrr galaxies with $850 \mu \mathrm{m}$ excess, the ratios are anticorrelated with the sSFR $\left(R_{\text {pear }}=-0.59\right)$ in the case of $\beta_{\mathrm{c}}=2$ (see the black triangles in the right panel). Although there is no trend of those ratios with sSFR in the case of free $\beta_{\mathrm{c}}$, the dIrr galaxies with $850 \mu \mathrm{m}$ excess have a relatively low sSFR and high $850 \mu \mathrm{m}$ observed-to-modeled flux ratios in our sample (see the red triangles in the right panel). These results suggest that the $850 \mu \mathrm{m}$ excess is easily detected in the dIrr galaxies with low sSFR. The lower the sSFR is, the stronger the $850 \mu \mathrm{m}$ excess emission is. In other words, there may be no presence of $850 \mu \mathrm{m}$ excess emission in the dIrr galaxies in which intense star formation is taking place.

\subsection{Cold Dust Temperature and Cold Dust Emissivity Index}

\subsubsection{The Influence of Submillimeter Data on $\mathrm{T}_{c}$}

Including more submillimeter data at wavelengths longward of $160 \mu \mathrm{m}$ (e.g., 500 or $850 \mu \mathrm{m}$ ) in SED fitting is necessary to accurately determine the dust temperature and masses of galaxies (Galametz et al. 2011, 2012; Rémy-Ruyer et al. 2015). It is needed to position the peak of dust SEDs accurately and sample the submillimeter slope of SEDs correctly (Galametz et al. 2011, 2012). In this work, we also perform the SED fitting using data up to $850 \mu \mathrm{m}$. Figure 11 in Appendix D shows the SEDs of our galaxies using this data coverage employing the TMBB model ( $\beta_{\mathrm{w}}=2$ and $\beta_{\mathrm{c}}=2$ or free) with the hierarchical Bayesian method.

Indeed, we find that the $T_{\mathrm{c}}$ obtained using submillimeter data longward of $160 \mu \mathrm{m}$ differ from those derived with data from $22-160 \mu \mathrm{m}$ only. We test the influence of including data longward of $160 \mu \mathrm{m}$ on the measured $T_{\mathrm{c}}$ of our galaxies. Those $T_{\mathrm{c}}$ values derived from SEDs using the multiple data ranges above are listed in Table 6 in Appendix C. We compare directly the $T_{\mathrm{c}}$ derived from the SED fitting with the data up to $250,350,500$, and $850 \mu \mathrm{m}$ to those derived with the data ranging from $22-160 \mu \mathrm{m}$. We find that for our galaxies $T_{\mathrm{c}}$ in the case of $\beta_{\mathrm{c}}=2$ obtained with data up to $250,350,500$, and $850 \mu \mathrm{m}$ differ from the $T_{\mathrm{c}}$ using data up to $160 \mu \mathrm{m}$ on average by $-3 \%,-6 \%,-9 \%$, and $-24 \%$, respectively. Figure 5 shows how $T_{\mathrm{c}}$ varies with submillimeter data at longer wavelengths added in the SED fitting in the case of $\beta_{\mathrm{c}}=2$. As more 

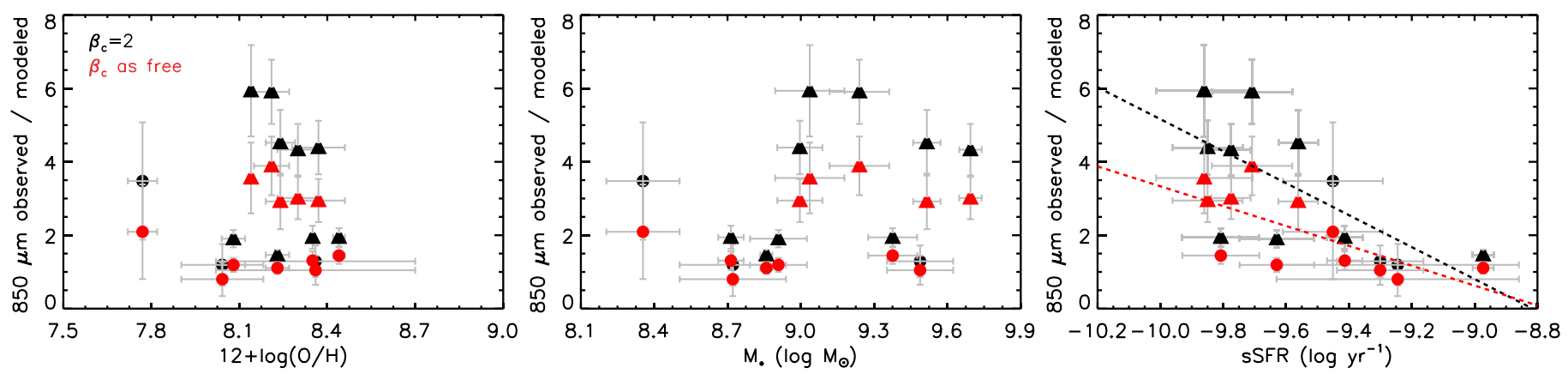

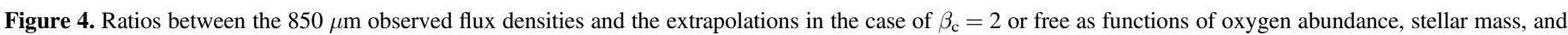
sSFR from left to right. For the convention on colors and symbols, see Figure 2.

submillimeter observations at longer wavelengths are added in the fits, the $T_{\mathrm{c}}$ derived decreases for most of our galaxies. As the number of submillimeter data increases, the better the peak of SED can be located, and the more accurately the cold dust temperature can be measured. The $T_{\mathrm{c}}$ derived from the SED fitting with data ranging from $22-850 \mu \mathrm{m}$ is the lowest for our galaxies except for UGC 05720, where the $T_{\mathrm{c}}$ derived with data up to $500 \mu \mathrm{m}$ is the lowest. The SED of this galaxy peaks at a shorter wavelength (about $50 \mu \mathrm{m}$ ) than that in the other galaxies (at $100 \mu \mathrm{m}<\lambda_{\text {peak }}<200 \mu \mathrm{m}$, see Figure 10 and Figure 11 in Appendix D). Therefore, for this galaxy, the wavelength of radiation dominating the cold dust components is shorter than that of other galaxies in our sample. For NGC 7694 and UGC 04305, there are no $T_{\mathrm{c}}$ derived by using data up to $160 \mu \mathrm{m}$ in the SED fitting due to no observation at $160 \mu \mathrm{m}$, which does not impact the trend of their $T_{\mathrm{c}}$ decreasing with increasing number of data points at longer submillimeter bands included in the fitting. However, the effect trend of submillimeter data on $T_{\mathrm{c}}$ is different when $\beta_{\mathrm{c}}$ is free. The $T_{\mathrm{c}}$ is the highest using data up to $850 \mu \mathrm{m}$ for free $\beta_{\mathrm{c}}$ because the $850 \mu \mathrm{m}$ data added tend to flatten the slope of SED at submillimeter bands. It may be due to the fact that some galaxies show $850 \mu \mathrm{m}$ excess. Above all, $850 \mu \mathrm{m}$ data is important to estimate cold dust temperature accurately for our sample.

\subsubsection{The Correlation between $\mathrm{T}_{c}$ and $\beta_{c}$}

Lamperti et al. (2019) showed that hierarchical Bayesian approach could reduce the degeneracy between $T_{\mathrm{c}}$ and $\beta_{\mathrm{c}}$ effectively. They show that there is an anticorrelation between $T_{\mathrm{c}}$ and $\beta_{\mathrm{c}}\left(R_{\text {pear }}=-0.52\right)$. We also derive the same anticorrelation between $T_{\mathrm{c}}$ and $\beta_{\mathrm{c}}\left(R_{\text {pear }}=-0.64\right)$ seen in Figure 6 using the same data range from $22-850 \mu \mathrm{m}$ as Lamperti et al. (2019). We note that in this work, we have applied a hierarchical approach with the aim to reduce the degeneracies between fitting parameters. It is possible that given the small sample size, the hierarchical approach has not be able to break this degeneracy completely. As an alternative, it is also possible that an intrinsic anticorrelation between $T_{\mathrm{c}}$ and $\beta_{\mathrm{c}}$ is present in this sample. In fact, we also plot the $\beta_{\mathrm{c}}$ as a function of $T_{\mathrm{c}}$ using the data in the $22-250 \mu \mathrm{m}$ range, in the $22-350 \mu \mathrm{m}$ range, and in the $22-500 \mu \mathrm{m}$ range (not shown here). There are positive correlations between $T_{\mathrm{c}}$ and $\beta_{\mathrm{c}}$ in these three wavelength ranges. However, these correlations may be artificially produced by the hierarchical SED fitting method. The values of $\beta_{\mathrm{c}}$ derived from SED fits using those three wavelength ranges are very similar. This may be an artificial effect. When $\beta_{\mathrm{c}}$ is free, in the three cases of fits up to 250,350 , and $500 \mu \mathrm{m}$, there may be not

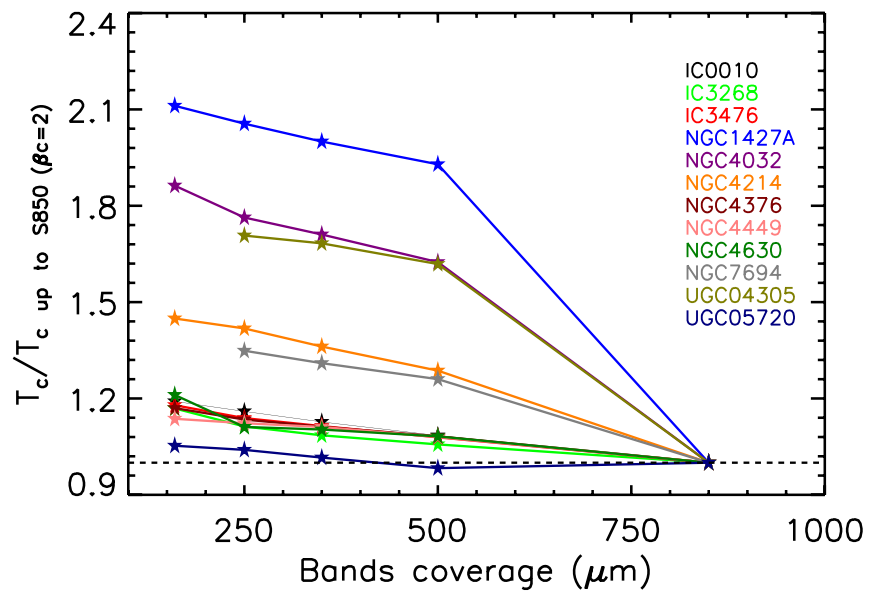

Figure 5. Variation of the cold dust temperature with submillimeter data coverage included in SED fitting (TMBB model with $\beta_{\mathrm{w}}=2$ and $\beta_{\mathrm{c}}=2$ ). Cold dust temperature are normalized to $T_{\mathrm{c}}$ obtained with the data coverage from $22-850 \mu \mathrm{m}$ (called " $T_{\mathrm{c}}$ up to $\mathrm{S} 850$ "). The $x$-axis describes the longest wavelength in the fitting. For example, "500" shows that using data range from $22-500 \mu \mathrm{m}$. The color for every galaxy is shown in the right top corner.

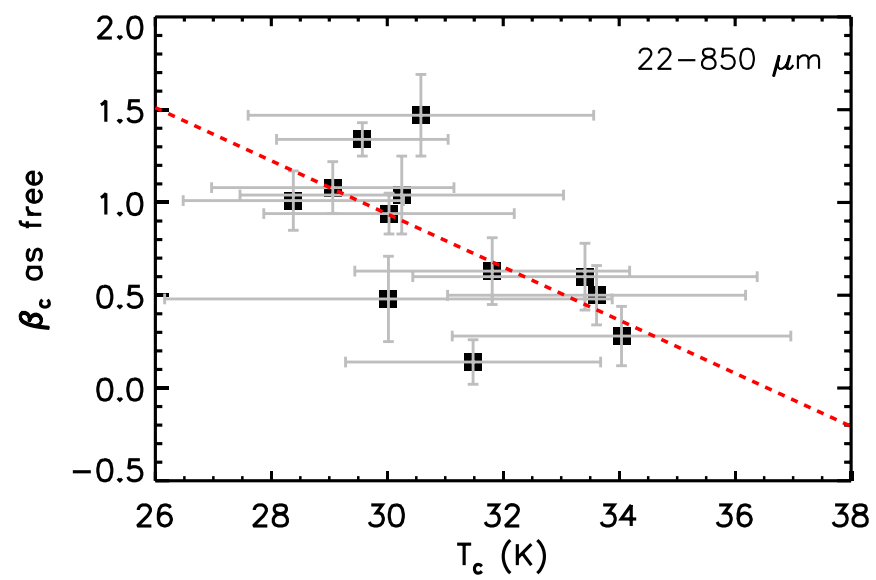

Figure 6. The relation between the cold dust temperature and cold emissivity index derived from the SED fits employing the TMBB model with $\beta_{\mathrm{w}}=2$ and $\beta_{\mathrm{c}}$ as free using data ranging from $22-850 \mu \mathrm{m}$. The gray lines show error bars. The red line fitted shows the trend of the both.

enough data points to constrain $T_{\mathrm{c}}$ and $\beta_{\mathrm{c}}$ well. In fact, we fit five to seven data points with the TMBB model, which has five free parameters $\left(T_{\mathrm{w}}, M_{\mathrm{w}}, T_{\mathrm{c}}, M_{\mathrm{c}}\right.$, and $\left.\beta_{\mathrm{c}}\right)$. Thus, the range of $T_{\mathrm{c}}$ and $\beta_{\mathrm{c}}$ are very small when we consider $\beta_{\mathrm{c}}$ as a free parameter in these three cases (see Table 6 in Appendix C). When we include the 

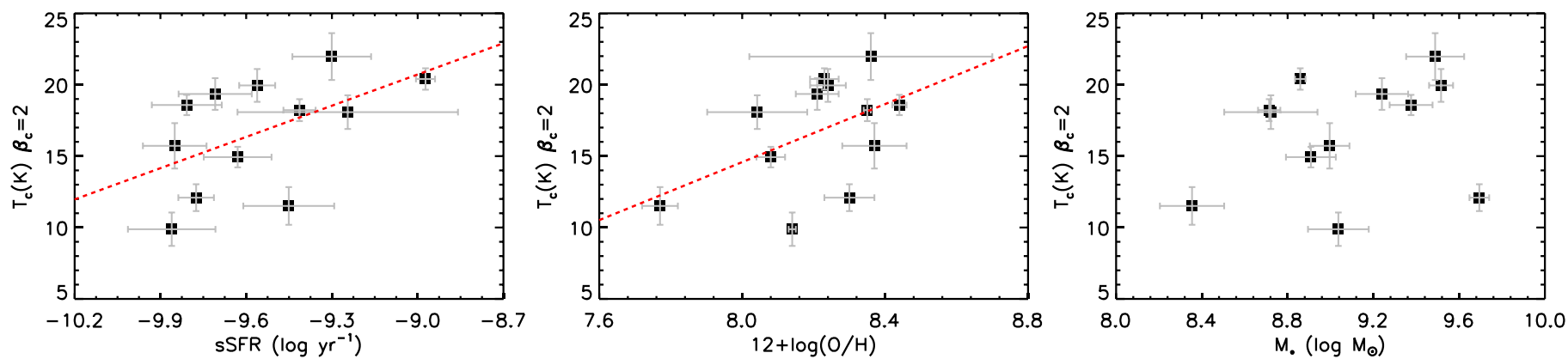

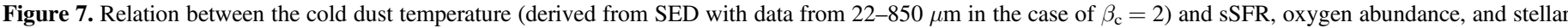
mass from left to right. The diagram is the same as Figure 6.

$850 \mu \mathrm{m}$ data, we can better constrain the $T_{\mathrm{c}}$ and $\beta_{\mathrm{c}}$. Although the values of $\beta_{\mathrm{c}}$ derived with data up to $850 \mu \mathrm{m}$ are smaller by $44 \%$ on average than the values of $\beta_{\mathrm{c}}$ derived from the SED fitting with data from $22-500 \mu \mathrm{m}$. This is due to the fact that the slope of the SED tends to become flatter when the $850 \mu \mathrm{m}$ flux is included in the fitting, which may be related to the presence of a submillimeter excess in some galaxies.

\subsubsection{The Relationships between Cold Dust Temperature and Fundamental Galaxy Parameters}

To study the correlation between $T_{\mathrm{c}}$ and fundamental galaxy parameters, we plot the $T_{\mathrm{c}}$ as functions of sSFR, oxygen abundance, and $M_{*}$ in the case of $\beta_{\mathrm{c}}=2$ in Figure 7 from left to right. In this section and Section 4.4.2, we analyze the properties of cold dust temperature and masses of our galaxies based on the TMBB model using $\beta_{\mathrm{c}}=2$ since the $\beta=2$ for MBB models is consistent with the results derived from standard dust model (Desert et al. 1990; Li \& Draine 2001; Relaño et al. 2018). The details are described in Section 3.2. For accuracy we adopt the $T_{\mathrm{c}}$ derived from the SED fitting with data $22-850 \mu \mathrm{m}$ (see Section 4.3.1 for details). We see that with the increase of sSFR, the $T_{\mathrm{c}}$ increases for our galaxies $\left(R_{\text {pear }}=0.52\right)$. This is consistent with the result of Rémy-Ruyer et al. (2015), who show the quantity $\sigma U$ (which is linked to the temperature) strongly correlates with the sSFR. This result also can be seen in Clemens et al. (2013), Cortese et al. (2014), and Lamperti et al. (2019). These suggest that higher star formation activity increases the average temperature of the cold dust component. There is a positive correlation between $T_{\mathrm{c}}$ and $12+\log (\mathrm{O} / \mathrm{H})$ for our sample $\left(R_{\text {pear }}=0.48\right)$. Only a rather weak relation between $T_{\mathrm{c}}$ and $M_{*}$ in our sample is found with $R_{\text {pear }}=0.25$. We may think that there is no relationship between them.

\subsection{Cold Dust Mass}

\subsubsection{The Influence of Submillimeter Data on $\mathrm{M}_{c}$}

The far-IR/submillimeter (longward of $160 \mu \mathrm{m}$ ) data is crucial to determine dust masses (Gordon et al. 2010; Galametz et al. 2011). In our sample, the $M_{\mathrm{c}}$ derived with submillimeter data do differ from the $M_{\mathrm{c}}$ derived using data only up to $160 \mu \mathrm{m}$. To show the influence of data longward of $160 \mu \mathrm{m}$ on the cold dust mass, we directly compare our dust masses derived with the data up to $250,350,500$, or $850 \mu \mathrm{m}$ to those derived with the data up to the $160 \mu \mathrm{m}$ constraint. These $M_{\mathrm{c}}$ measured are all listed in Table 6 in Appendix C. The $M_{\mathrm{c}}$ derived by the SED fitting using the data up to $850 \mu \mathrm{m}$ or at least $500 \mu \mathrm{m}$ are the highest in the case of $\beta_{\mathrm{c}}=2$. Figure 8

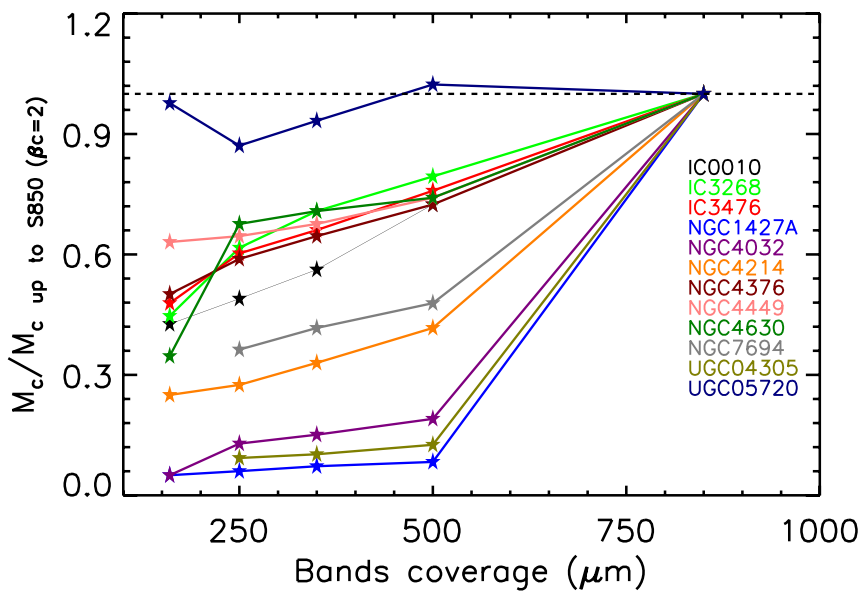

Figure 8. Variation of the cold dust mass with submillimeter data coverage included in SED fitting (TMBB model with $\beta_{\mathrm{w}}=2$ and $\beta_{\mathrm{c}}=2$ ). Cold dust masses are normalized to $M_{\mathrm{c}}$ obtained with the data coverage from $22-850 \mu \mathrm{m}$ (called " $M_{\mathrm{c}}$ up to S850"). The $x$-axis describes the longest wavelength in the fitting. For example, "500" shows that using data range from $22-500 \mu \mathrm{m}$. The color for every galaxy is shown in the right bottom.

shows how the cold dust masses vary with the submillimeter coverage included in fitting in the case of $\beta_{\mathrm{c}}$ fixed to 2 . The $M_{\mathrm{c}}$ derived show a trend of increase when more submillimeter data at longer wavelengths were included in the fitting, except for UGC $05720 . M_{\mathrm{c}}$ of UGC 05720 also increase with the addition of number of SPIRE bands and its $M_{\mathrm{c}}$ is the highest when using data up to $500 \mu \mathrm{m}$. For the other galaxies, the $M_{\mathrm{c}}$ derived from fits with data up to $850 \mu \mathrm{m}$ is the highest. Similarly to the effect we observed on $T_{\mathrm{c}}$, this may be due to the fact that the peak wavelength (at about $50 \mu \mathrm{m}$ ) of UGC 05720 is shorter than that of the other galaxies (at $100<\lambda_{\text {peak }}<200 \mu \mathrm{m}$ ). For NGC 7694 and UGC 04305, there is no $M_{\mathrm{c}}$ derived from 22-160 $\mu \mathrm{m}$ SED fitting due to no observation at $160 \mu \mathrm{m}$. But $M_{\mathrm{c}}$ of them increase with addition of data at longer submillimeter bands. These behaviors in the case of $\beta_{\mathrm{c}}=2$ are more in line with the general view that the higher dust mass are derived using submillimeter data than dust mass derived not using submillimeter data for metal-poor galaxies due to the flattening of the submillimeter slope (Galametz et al. 2012). Galametz et al. (2012) also shows that the cold dust mass derived using the complete SPIRE data are good to obtain the dust mass in the case of fixed $\beta_{\mathrm{c}}$. They do not test the influence of $850 \mu \mathrm{m}$ data on the $M_{\mathrm{c}}$. Above all, using submillimeter data included in fitting (such as data up to $850 \mu \mathrm{m}$ or at least up to $500 \mu \mathrm{m}$ ) is necessary to get an most accurate estimation of the $M_{\mathrm{c}}$ in the case of fixed $\beta_{\mathrm{c}}=2$. However, the effect of submillimeter wavelengths coverage on cold dust mass is different when $\beta_{\mathrm{c}}$ is 

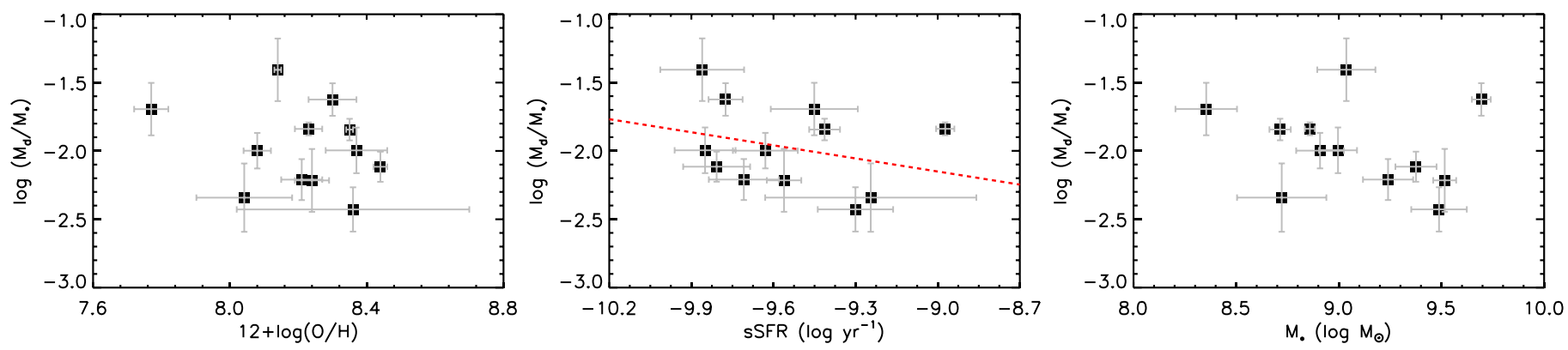

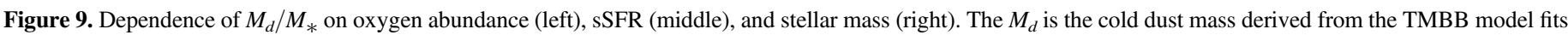
with $\beta_{\mathrm{c}}=2$ applying a hierarchical Bayesian method with the data from $22-850 \mu \mathrm{m}$. The diagram is the same as Figure 6 .

left free to vary. The behavior of cold dust mass is different for each galaxy with increase of number of data at submillimeter bands (see Table 6 in Appendix C). The cold dust mass we obtained using data up to SPIRE bands or $850 \mu \mathrm{m}$ is still different from those derived with data stopping at $160 \mu \mathrm{m}$.

\subsubsection{The Relationships between the Cold Dust-to-stellar Mass Ratio and Fundamental Galaxy Parameters}

In this section, we consider the dust-to-stellar mass ratio $\left(M_{d} / M_{*}\right)$ and its relation with fundamental galaxy parameters. Past studies suggest that $M_{d} / M_{*}$ has a strong correlation with sSFR (da Cunha et al. 2010; Skibba et al. 2011; De Vis et al. 2017), but weak anticorrelation with $M_{*}$ (Skibba et al. 2011; Bourne et al. 2012; De Vis et al. 2017), or increases with the decrease of metallicity (Skibba et al. 2011). We plot the cold dust-to-stellar mass ratio as a function of oxygen abundance (left panel), sSFR (middle panel), and stellar mass (right panel) of our galaxies in the case of $\beta_{\mathrm{c}}=2$ in Figure 9. For accuracy we use the $M_{\mathrm{c}}$ derived from the SED fitting with data ranging from 22-850 $\mu \mathrm{m}$ (see Section 4.4.1 for details). Since the warm dust mass are only $0.007 \%-0.400 \%$ of cold dust mass using this coverage of data in the case of $\beta_{\mathrm{c}}=2$, we use the $M_{\mathrm{c}}$ as the total dust mass $\left(M_{d}\right)$ directly (Galametz et al. 2012). Similar to the properties of cold dust temperature analyzed in Section 4.3.3, we analyze the above relation in the case of $\beta_{\mathrm{c}}=2$ since this case is the model using dust grains with standard properties (Galametz et al. 2014). We find that $M_{d} / M_{*}$ decrease weakly with the increase of sSFR for our galaxies $\left(R_{\text {pear }}=-0.29\right)$. This is different from previous results mentioned above reporting a strong correlation between $M_{d} / M_{*}$ and sSFR. A weak anticorrelation between $M_{d} / M_{*}$ and oxygen abundance is found with $R_{\text {pear }}=-0.25$ in the sample. We can think that there is no relationship between them. This is also true for the correlation between $M_{d} / M_{*}$ and $M_{*}$ for our galaxies $\left(R_{\text {pear }}=-0.24\right)$. Perhaps our sample is not large enough to show a strong trend. We need more nearby dIrr galaxies to test these trends.

\section{Summary and Conclusions}

We combine WISE, IRAS, Spitzer, Herschel, and Planck data to model the SED of 12 nearby dIrr galaxies with a TMBB model in the case of $\beta_{\mathrm{c}}=2$ or $\beta_{\mathrm{c}}$ as free using a hierarchical Bayesian method. Based on the TMBB model with $\beta_{\mathrm{w}}=2$ and $\beta_{\mathrm{c}}=2$, we probe the potential submillimeter excess emission at 350,500 , and $850 \mu \mathrm{m}$. And we analyze which situation could be likely to promote the existence of $850 \mu \mathrm{m}$ excess emission. The main conclusions are as follow:
1. No galaxy shows excess emission at $350 \mu \mathrm{m}$. One galaxy (NGC 4214) shows an excess emission at $500 \mu \mathrm{m}$. Nine galaxies (IC 3268, IC 3476, NGC 1427A, NGC 4032, NGC 4214, NGC 4376, NGC 4449, NGC 4630, and NGC 7694) show $850 \mu \mathrm{m}$ excess emission, and five of them (IC 3268, NGC 1427A, NGC 4032, NGC 4376, and NGC 7694) still exhibit a clear $850 \mu \mathrm{m}$ excess for free $\beta_{\mathrm{c}}$.

2. We confirm that the excess emission at $850 \mu \mathrm{m}$ are not accounted for by non-dust contributions $(\mathrm{CO}(3-2)$ line, synchrotron and free-free emission) for our dIrr galaxies. But for NGC 1427A, we do not know if the synchrotron and free-free emission cause its $850 \mu \mathrm{m}$ excess.

3. For our dIrr galaxies, the $850 \mu \mathrm{m}$ observed-to-modeled flux ratio increases with atomic hydrogen gas mass fraction and decreases with molecular hydrogen gas mass fraction. The $850 \mu \mathrm{m}$ excess emission is more likely to be detected in the dIrr galaxies with high atomic hydrogen gas mass fraction or low molecular hydrogen gas mass fraction. The smaller the molecular hydrogen gas mass fraction is, the stronger the $850 \mu \mathrm{m}$ excess is.

4. There is no connection between the $850 \mu \mathrm{m}$ observed-tomodeled flux ratio and cold dust mass and cold dust temperature for our dIrr galaxies. As the ratio between cold dust mass and gas mass $\left(M_{\mathrm{c}} / M_{\mathrm{gas}}\right)$ decreases, the $850 \mu \mathrm{m}$ observed-to-modeled flux ratio tends to increase for our galaxies. The $850 \mu \mathrm{m}$ excess emission is more likely to occur in the dIrr galaxies with low $M_{\mathrm{c}} / M_{\mathrm{gas}}$. And the lower the $M_{\mathrm{c}} / M_{\mathrm{gas}}$ is, the stronger the $850 \mu \mathrm{m}$ excess is.

5. The $850 \mu \mathrm{m}$ observed-to-modeled flux ratio does not show a particular trend with the oxygen abundance for our galaxies. There is a rather weak correlation between the $850 \mu \mathrm{m}$ observed-to-modeled flux ratio and stellar mass for our galaxies. The $850 \mu \mathrm{m}$ observed-to-modeled flux ratio is strongly anticorrelated with sSFR for our dIrr galaxies. The $850 \mu \mathrm{m}$ excess emission is not related to metallicity and seems to be independent of the stellar mass for our dIrr galaxies. The $850 \mu \mathrm{m}$ excess is easily detected in the dIrr galaxies with low sSFR. The lower the sSFR is, the stronger the $850 \mu \mathrm{m}$ excess is.

In this work, we also derive the cold dust parameters $\left(T_{\mathrm{c}}, M_{\mathrm{c}}\right.$, and $\beta_{\mathrm{c}}$ ) from SED fitting using different data coverage of $22-160 \mu \mathrm{m}, 22-250 \mu \mathrm{m}, 22-350 \mu \mathrm{m}, 22-500 \mu \mathrm{m}$, and $22-850 \mu \mathrm{m}$ with the same technique. We test the influence that including observations at wavelength $>160 \mu \mathrm{m}$ in the SED fitting on the measured $T_{\mathrm{c}}$ and $M_{\mathrm{c}}$, discuss the distribution of $T_{\mathrm{c}}$ with $\beta_{\mathrm{c}}$ and study the different correlation between cold dust 
parameters $\left(T_{\mathrm{c}}\right.$ and $\left.M_{d} / M_{*}\right)$ and fundamental galaxy properties. The main findings are as follows:

1. Generally, including submillimeter data in the SED fitting (at least up to $500 \mu \mathrm{m}$ ) in the case of $\beta_{\mathrm{c}}=2$ allows us to estimate cold dust temperature and cold dust mass more accurately.

2. The cold dust temperature $T_{\mathrm{c}}$ correlates with $\mathrm{sSFR}$ and oxygen abundance for our galaxies.

3. The cold dust-to-stellar mass ratio $\left(M_{d} / M_{*}\right)$ is weakly anticorrelated with sSFR for our galaxies.

Considering the limited number of galaxies in our sample, we need more nearby dIrr galaxies to test our results.

This work was mainly funded by the open program of the Key Laboratory of Xinjiang Uygur Autonomous Region under grant No. 2019D04023 and the National Natural Science foundation of China (NSFC) under grant No. 11973076. It was also partially funded by the NSFC under grant Nos. 11433008 and 11903070 , by the "TianShan Youth Plan" under grant No. 2018Q084, by the Project of Xinjiang Uygur Autonomous Region of China for Flexibly Fetching in Upscale Talents, by the Heaven Lake Hundred Talent Program of Xinjiang Uygur Autonomous Region of China, by the CAS "Light of West China" Program 2020-XBQNXZ-017 and by the CAS Light of West China Program under grant No. 2018-XBQNXZ-B-024. I.L. acknowledges support from the Comunidad de Madrid through the Atracción de Talento Investigador Grant 2018-T1/TIC-11035. Zeming Zhou thanks for the Cultivation Project for LAMOST Scientific Payoff and Research Achievement of CAMS-CAS. J.J. Q. thanks the support of NSFC (No.12003080), the 871 Guangdong Basic and Applied Basic Research Foundation (No. 2019A1515110588), and the Fundamental Research Funds for the Central Universities, Sun Yat-sen University (No. 19284). M.Z. thanks for the Doctoral Scientific Research Foundation of Shangrao Normal University (grant No. K6000449).

DustPedia is a collaborative focused research project supported by the European Union under the Seventh Framework Programme (2007-2013) call (proposal No. 606847). The participating institutions are Cardiff University, UK; National Observatory of Athens, Greece; Ghent University, Belgium; Université Paris Sud, France; National Institute for Astrophysics, Italy and CEA, France. Herschel is an ESA space observatory with science instruments provided by Europeanled Principal Investigator consortia and with important participation from NASA. PACS has been developed by a consortium of institutes led by MPE (Germany) and including UVIE (Austria); KU Leuven, CSL, IMEC (Belgium); CEA, LAM (France); MPIA (Germany); INAF-IFSI/OAA/OAP/ OAT, LENS, SISSA (Italy); IAC (Spain). This development has been supported by the funding agencies BMVIT (Austria), ESA-PRODEX (Belgium), CEA/CNES (France), DLR (Germany), ASI/INAF (Italy), and CICYT/MCYT (Spain). SPIRE has been developed by a consortium of institutes led by Cardiff University (UK) and including University of Lethbridge (Canada); NAOC (China); CEA, LAM (France); IFSI, University of Padua (Italy); IAC (Spain); Stockholm Observatory (Sweden); Imperial College London, RAL, UCL-MSSL, UKATC, University of Sussex (UK); and Caltech, JPL, NHSC, University of Colorado (USA). This development has been supported by national funding agencies: CSA (Canada); NAOC (China); CEA, CNES, CNRS (France); ASI (Italy); MCINN
(Spain); SNSB (Sweden); STFC, UKSA (UK); and NASA (USA). This work also makes use of data obtained with the Planck Space Telescope (http://www.esa.int/Planck) (an ESA science mission with instruments and contributions directly funded by ESA Member States, NASA, and Canada), the Spitzer Space Telescope (which is operated by the Jet Propulsion Laboratory, California Institute of Technology under a contract with NASA), and the WISE space telescope. (which is a joint project of the University of California, Los Angeles, and the Jet Propulsion Laboratory/California Institute of Technology, and NEOWISE, which is a project of the Jet Propulsion Laboratory/California Institute of Technology. WISE and NEOWISE are funded by the National Aeronautics and Space Administration.)

Software: Stan (Carpenter et al. 2017), PyStan (Stan Development Team 2018).

\section{Appendix A \\ Non-dust Contribution to the $850 \mu \mathrm{m}$ Flux Densities}

There are very few observations of $\mathrm{CO}$ for our sample since $\mathrm{CO}$ observation of dwarf galaxies is difficult (Rémy-Ruyer et al. 2013). Although there are CO maps of Planck data (Planck Collaboration et al. 2016a), they rely on the velocity range of the Milky Way (Tibbs et al. 2018). Since the velocities of most galaxies in our sample are large with respect to the velocity range of the Milky Way (see Table 1), we should not use the published Planck CO maps to estimate $\mathrm{CO}$ emission for our galaxies. The same is true for M33 (see Section 3.3 in Tibbs et al. 2018) with velocity of $-179 \mathrm{~km} \mathrm{~s}^{-1}$, which is substantially different to the velocity range of the Milky Way applied to producing Planck CO maps. We also checked the maps of Planck $\mathrm{CO}$ emission then determined that they are not suitable for our analysis in this work. So we had to estimate the contribution from the $\mathrm{CO}(3-2)$ line at $850 \mu \mathrm{m}$ indirectly. We calculate the luminosity at $12 \mu \mathrm{m}$ for each galaxy with their $12 \mu \mathrm{m}$ flux obtained from Clark et al. (2018), then get the total molecular hydrogen gas mass, $\log \left(M_{\mathrm{H}_{2}}\right)$, using the formula (Jiang et al. 2015)

$$
\begin{aligned}
\log M_{\mathrm{H}_{2}}\left(\mathrm{CO}_{1-0}\right)= & (0.88 \pm 0.03) \log L_{12 \mu \mathrm{m}} \\
& +(1.49 \pm 0.27) .
\end{aligned}
$$

The values of $\log \left(M_{\mathrm{H}_{2}}\right)$ for our sample are listed in Table 1. The $M_{\mathrm{H}_{2}}\left(\mathrm{CO}_{1-0}\right)$ is calculated by $M_{\mathrm{H}_{2}}=\alpha_{\mathrm{CO}} L_{\mathrm{CO} 10}$, where $\alpha_{\mathrm{CO}}$ is the $\mathrm{CO}(1-0)$-to- $\mathrm{H}_{2}$ conversion factor. Considering that $\alpha_{\mathrm{CO}}$ varies with metallicity (Shi et al. 2016; Accurso et al. 2017), we use the following relation (Accurso et al. 2017) to derive $\alpha_{\mathrm{CO}}$ for each galaxy:

$$
\begin{gathered}
\log \alpha_{\mathrm{CO}}( \pm 0.165 \mathrm{dex})=14.752-1.623 \\
\quad \times[12+\log (\mathrm{O} / \mathrm{H})]+0.062 \log \Delta(\mathrm{MS})
\end{gathered}
$$

with the distance from the main sequence, $\Delta(\mathrm{MS})=$ $\frac{\mathrm{sSFR}_{\text {measured }}}{\operatorname{sSFR}_{\mathrm{ms}}\left(z, M_{*}\right)} \cdot \mathrm{sSFR}_{\text {measured }}$ denotes specific SFR of our galaxies calculated by ratio between SFR and stellar mass (listed in Table 1). The $\operatorname{SSFR}_{\mathrm{ms}}\left(z, M_{*}\right)$ is related to redshift $(z)$ and stellar mass $\left(M_{*}\right)$ by the equation (Accurso et al. 2017):

$$
\begin{gathered}
\log \left(\operatorname{sSFR}_{\mathrm{ms}}\left(z, M_{*}\right)\right)=-1.12+1.14 z-0.19 z^{2} \\
-(0.3+0.13 z) \times\left(\log M_{*}-10.5\right)\left(\mathrm{Gyr}^{-1}\right) .
\end{gathered}
$$

The derived $\alpha_{\mathrm{CO}}$ in units of $M_{\odot}\left(\mathrm{K} \mathrm{km} \mathrm{s}^{-1} \mathrm{pc}^{2}\right)^{-1}$ are listed in Table 2 in Appendix A. In this way, we derive the luminosity of $\mathrm{CO}(1-0)$ line, $L_{\mathrm{CO} 10}$. Then, we derive the intensity of 
Table 2

The Flux of the Planck $850 \mu \mathrm{m}, \mathrm{CO}(3-2)$ Line and Free-Free + Synchrotron at $850 \mu \mathrm{m}$ in Units of Jansky and $\mathrm{CO}(1-0)$-to- $\mathrm{H}_{2} \mathrm{Conversion} \mathrm{Factor} \mathrm{in} \mathrm{Units}$ of $M_{\odot}\left(\mathrm{K} \mathrm{km} \mathrm{s}^{-1} \mathrm{pc}^{2}\right)^{-1}$

\begin{tabular}{lcccc}
\hline \hline Galaxy & $S_{850}$ & $S_{\mathrm{CO}(3-2)}$ & $S_{\text {free-free+synchrotron }}$ & $\alpha_{\mathrm{CO}}$ \\
\hline IC 0010 & $5.81 \pm 1.36$ & 0.0182 & 0.0934 & $53.01 \pm 20.14$ \\
IC 3268 & $0.12 \pm 0.02$ & 0.0004 & 0.0020 & $24.81 \pm 9.42$ \\
IC 3476 & $0.15 \pm 0.02$ & 0.0020 & 0.0053 & $\ldots$ \\
NGC 1427A & $0.13 \pm 0.01$ & 0.0002 & 0.0020 & $16.24 \pm 6.17$ \\
NGC 4032 & $0.22 \pm 0.02$ & 0.0008 & 0.0150 & $19.37 \pm 7.36$ \\
NGC 4214 & $1.25 \pm 0.08$ & 0.0059 & 0.0002 & $43.54 \pm 16.54$ \\
NGC 4376 & $0.18 \pm 0.02$ & 0.0004 & 0.0330 & $26.85 \pm 10.20$ \\
NGC 4449 & $1.36 \pm 0.08$ & 0.0078 & 0.0016 & $11.27 \pm 10.35$ \\
NGC 4630 & $0.20 \pm 0.02$ & 0.0011 & 0.0020 & $14.31 \pm 5.28$ \\
NGC 7694 & $0.19 \pm 0.02$ & 0.0001 & 0.0015 & $138.93 \pm 52.78$ \\
UGC 04305 & $0.26 \pm 0.02$ & 0.0029 & 0.0061 & $16.42 \pm 6.24$ \\
UGC 05720 & $0.06 \pm 0.02$ & & & \\
\hline
\end{tabular}

Note. The Planck $850 \mu \mathrm{m}$ fluxes are not corrected for non-dust contribution. NGC 1427A have no available mid-frequency radio flux to extrapolate free-free and synchrotron emission at $850 \mu \mathrm{m}$.

$\mathrm{CO}(1-0)$ line in units of jansky kilometer per second, $S_{\mathrm{CO} 10} \Delta V$, by using the formula (Solomon et al. 1997)

$$
L_{\mathrm{CO} 10}=3.25 \times 10^{7} S_{\mathrm{CO} 10} \Delta V \nu_{\mathrm{obs}}^{-2} D_{L}^{2}(1+z)^{-3}
$$

with the $\nu_{\mathrm{obs}}$ observing frequency of $\mathrm{CO}(1-0)$ line of $115 \mathrm{GHz}$ and the $D_{L}$ luminosity distance in Mpc derived from $D_{L}=D(1+z)$ (Mao et al. 2010). $D$ is the best distance of a galaxy listed in Table 1. Finally, to derive the flux densities of the $\mathrm{CO}(3-2)$ line, we use the following relation (Hermelo et al. 2016):

$$
S=R_{31} \times\left(\frac{\nu}{115}\right)^{2}\left(S_{\mathrm{CO} 10} \Delta V\right),
$$

where $S$ is the flux density of the $\mathrm{CO}(3-2)$ line, and $R_{31}$ is the CO line ratio $R_{31}=I_{32} / I_{10}$. We adopt a typical value $R_{31}=0.55$ (Lamperti et al. 2020). The $\nu$ is the observing frequency of the $\mathrm{CO}(3-2)$ line of $345 \mathrm{GHz}$. The Planck $850 \mu \mathrm{m}$ filter has a width of $101.4 \mathrm{GHz}\left(85,032 \mathrm{~km} \mathrm{~s}^{-1}\right)$ and transmission close to $100 \%$ at the frequency of the $\mathrm{CO}(3-2)$ line. The fluxes of the $\mathrm{CO}(3-2)$ line we derived are listed in Table 2 in Appendix A. The $\mathrm{CO}(3-2)$ line emission contributes from $0.04 \%-4.83 \%$ to the total $850 \mu \mathrm{m}$ flux for our galaxies. We ignore the error of the $\mathrm{CO}(3-2)$ line flux, since their values are smaller than the uncertainties of the $850 \mu \mathrm{m}$ flux densities (see Table 2).

To estimate the contribution from synchrotron and free-free emission at $850 \mu \mathrm{m}$, following Chang et al. 2020 we searched for some fluxes of mid-frequency radio continuum emission ranging from $1.4-10.7 \mathrm{GHz}$ first from NED, which are mainly contributed by free-free and synchrotron emission (see Figure 1 of Condon (1992)). Then we combine two power laws,
$S_{v} \propto v^{-\alpha}$ with radio spectral indexes $\alpha=0.1$ for free-free emission and $\alpha=0.8$ for synchrotron emission (Condon 1992; Niklas et al. 1997) to fit the above mid-frequency radio fluxes in order to extrapolate radio flux at $850 \mu \mathrm{m}$. The synchrotron and free-free emission contribute from $0.1-10 \%$ to the total $850 \mu \mathrm{m}$ flux for our galaxies except for NGC 1427A. These contributions at $850 \mu \mathrm{m}$ are listed in Table 2 in Appendix A. Those values are less than the uncertainties of $850 \mu \mathrm{m}$ flux (see Table 2). So we ignore the error of synchrotron and free-free emission. For NGC 1427A, we did not find the available flux at those mid-frequency radio wavelengths, so the contamination of synchrotron and free-free emission at $850 \mu \mathrm{m}$ is not removed.

For the uncertainty of $850 \mu \mathrm{m}$ corrected flux, we ignore the error of $\mathrm{CO}(3-2)$ line, synchrotron and free-free emission due to their smaller values than the uncertainty of $850 \mu \mathrm{m}$ flux. The fluxes at $850 \mu \mathrm{m}$ corrected for $\mathrm{CO}(3-2)$ line, synchrotron and free-free emission with their uncertainties are listed in the table 5 in Appendix B.

\section{Appendix B}

\section{Observations and Predictions at 350, 500, and $850 \mu \mathrm{m}$}

In this section, Tables 3-5 give the observed flux densities and modeled flux densities at 350,500 , and $850 \mu \mathrm{m}$ using the TMBB model with $\beta_{\mathrm{w}}=2$ and $\beta_{\mathrm{c}}=2$ or $\beta_{\mathrm{c}}$ as free and applying hierarchical Bayesian techniques, where the $850 \mu \mathrm{m}$ observed flux have been corrected for non-dust emission $\mathrm{CO}(3$ -2) line emission, free-free and synchrotron emission). Table 5 also gives a comparison of observations and predictions at $850 \mu \mathrm{m}$ as $\frac{850 \text { obs corrected }}{850 \text { modeled }}$ in the case of $\beta_{\mathrm{c}}=2$ and $\beta_{\mathrm{c}}$ as free. 
Table 3

The $350 \mu \mathrm{m}$ Observations and Predictions

\begin{tabular}{|c|c|c|c|c|}
\hline \multirow{2}{*}{ Galaxy } & \multirow{2}{*}{$\begin{array}{c}F_{\nu}(350 \mu \mathrm{m}) \\
(\mathrm{Jy})\end{array}$} & \multirow{2}{*}{$\begin{array}{c}\beta_{\mathrm{c}}=2 \\
350 \text { modeled }(\mathrm{Jy})\end{array}$} & \multicolumn{2}{|c|}{$\beta_{\mathrm{c} \text { free }}$} \\
\hline & & & Values & 350 modeled $(\mathrm{Jy})$ \\
\hline IC 0010 & $52.93 \pm 40.42$ & $62.66_{-47.37}^{+101.72}$ & $1.30 \pm 0.48$ & $73.48_{-56.90}^{+144.86}$ \\
\hline IC 3268 & $0.53 \pm 0.08$ & $0.39_{-0.11}^{+0.12}$ & $1.40 \pm 0.41$ & $0.43_{-0.19}^{+0.24}$ \\
\hline IC 3476 & $1.21 \pm 0.11$ & $1.08_{-0.25}^{+0.27}$ & $1.25 \pm 0.47$ & $1.20_{-0.44}^{+0.57}$ \\
\hline NGC 1427A & $0.40 \mp 0.09$ & $0.29_{-0.13}^{+0.17}$ & $1.15 \pm 0.60$ & $0.34_{-0.18}^{+0.31}$ \\
\hline NGC 4032 & $0.86 \pm 0.12$ & $0.64_{-0.20}^{+0.22}$ & $1.29 \pm 0.51$ & $0.75_{-0.37}^{+0.59}$ \\
\hline NGC 4214 & $10.72 \pm 0.89$ & $8.58_{-1.86}^{+2.26}$ & $1.31 \pm 0.41$ & $9.54_{-3.87}^{+4.63}$ \\
\hline NGC 4376 & $0.57 \pm 0.08$ & $0.47_{-0.13}^{+0.13}$ & $1.38 \pm 0.42$ & $0.56_{-0.25}^{+0.25}$ \\
\hline NGC 4449 & $15.29 \pm 1.02$ & $15.09_{-3.19}^{+3.53}$ & $1.51 \pm 0.38$ & $16.59_{-6.17}^{+6.86}$ \\
\hline NGC 4630 & $1.67 \pm 0.17$ & $1.58_{-0.33}^{+0.50}$ & $1.22 \pm 0.48$ & $1.84_{-0.76}^{+1.04}$ \\
\hline NGC 7694 & $0.74 \pm 0.08$ & $0.62_{-0.19}^{+0.18}$ & $1.30 \pm 0.49$ & $0.72_{-0.28}^{+0.37}$ \\
\hline UGC 04305 & $1.13 \pm 0.68$ & $0.95_{-0.68}^{+1.31}$ & $1.10 \pm 0.55$ & $1.09_{-0.81}^{+1.87}$ \\
\hline UGC 05720 & $0.70 \pm 0.70$ & $0.67_{-0.22}^{+0.28}$ & $1.63 \pm 0.43$ & $0.71_{-0.36}^{+0.48}$ \\
\hline
\end{tabular}

Note. The SED fits using the data coverage from $22-250 \mu \mathrm{m}$.

Table 4

The $500 \mu \mathrm{m}$ Observations and Predictions

\begin{tabular}{|c|c|c|c|c|}
\hline \multirow[t]{2}{*}{ Galaxy } & \multirow{2}{*}{$\begin{array}{c}F_{\nu}(500 \mu \mathrm{m}) \\
(\mathrm{Jy})\end{array}$} & \multirow{2}{*}{$\begin{array}{c}\beta_{\mathrm{c}}=2 \\
500 \text { modeled }(\mathrm{Jy})\end{array}$} & \multicolumn{2}{|c|}{$\beta_{\mathrm{c} \text { free }}$} \\
\hline & & & Values & 500 modeled (Jy) \\
\hline IC 0010 & $21.08 \pm 14.08$ & $23.73_{-16.88}^{+43.89}$ & $1.31 \pm 0.30$ & $32.27_{-26.86}^{+63.18}$ \\
\hline IC 3268 & $0.23 \pm 0.05$ & $0.15_{-0.04}^{+0.05}$ & $1.50 \pm 0.27$ & $0.17_{-0.06}^{+0.12}$ \\
\hline IC 3476 & $0.49 \pm 0.05$ & $0.40_{-0.08}^{+0.10}$ & $1.38 \pm 0.24$ & $0.48_{-0.17}^{+0.25}$ \\
\hline NGC 1427A & $0.17 \mp 0.05$ & $0.12_{-0.05}^{+0.08}$ & $1.29 \pm 0.30$ & $0.15_{-0.08}^{+0.13}$ \\
\hline NGC 4032 & $0.36 \pm 0.04$ & $0.25_{-0.08}^{+0.13}$ & $1.41 \pm 0.24$ & $0.30_{-0.12}^{+0.24}$ \\
\hline NGC 4214 & $5.02 \pm 0.47$ & $3.31_{-0.73}^{+0.92}$ & $1.42 \pm 0.25$ & $4.00_{-1.27}^{+2.40}$ \\
\hline NGC 4376 & $0.23 \pm 0.05$ & $0.17_{-0.04}^{+0.05}$ & $1.47 \pm 0.23$ & $0.21_{-0.10}^{+0.13}$ \\
\hline NGC 4449 & $6.00 \pm 0.48$ & $5.02_{-0.76}^{+1.09}$ & $1.67 \pm 0.22$ & $5.75_{-1.78}^{+2.30}$ \\
\hline NGC 4630 & $0.58 \pm 0.06$ & $0.57_{-0.11}^{+0.18}$ & $1.37 \pm 0.22$ & $0.69_{-0.25}^{+0.31}$ \\
\hline NGC 7694 & $0.30 \pm 0.04$ & $0.23_{-0.06}^{+0.06}$ & $1.44 \pm 0.26$ & $0.28_{-0.10}^{+0.20}$ \\
\hline UGC 04305 & $0.63 \pm 0.43$ & $0.37_{-0.26}^{+0.77}$ & $1.30 \pm 0.39$ & $0.46_{-0.38}^{+1.34}$ \\
\hline UGC 05720 & $0.29 \pm 0.05$ & $0.23_{-0.06}^{+0.09}$ & $1.76 \pm 0.33$ & $0.23_{-0.10}^{+0.17}$ \\
\hline
\end{tabular}

Note. The SED fits using the data coverage from $22-350 \mu \mathrm{m}$.

Table 5

Comparison of $850 \mu \mathrm{m}$ Observations and Predictions

\begin{tabular}{|c|c|c|c|c|c|c|}
\hline \multirow{2}{*}{ Galaxy } & \multirow{2}{*}{$\begin{array}{c}F_{\nu}(850 \mu \mathrm{m}) \\
(\mathrm{Jy})\end{array}$} & \multicolumn{2}{|c|}{$\beta_{\mathrm{c}}=2$} & \multicolumn{3}{|c|}{$\beta_{\mathrm{c} \text { free }}$} \\
\hline & & 850 modeled $(\mathrm{Jy})$ & $\frac{850 \text { obs corrected }}{850 \text { modeled }}$ & values & 850 modeled (Jy) & $\frac{850 \text { obs corrected }}{850 \text { modeled }}$ \\
\hline IC 0010 & $5.70 \pm 1.36$ & $4.78_{-3.37}^{+7.67}$ & $1.19 \pm 0.57$ & $1.32 \pm 0.22$ & $7.10_{-6.28}^{+18.85}$ & $0.80 \pm 0.46$ \\
\hline IC 3268 & $0.12 \pm 0.02$ & $0.03_{-0.01}^{+0.01}$ & $4.52 \pm 0.89$ & $1.40 \pm 0.17$ & $0.04_{-0.02}^{+0.03}$ & $2.92 \pm 0.76$ \\
\hline IC 3476 & $0.14 \pm 0.02$ & $0.07_{-0.01}^{+0.02}$ & $1.94 \pm 0.32$ & $1.35 \pm 0.16$ & $0.11_{-0.04}^{+0.07}$ & $1.31 \pm 0.25$ \\
\hline NGC1427A & $0.13 \pm 0.01$ & $0.02_{-0.01}^{+0.02}$ & $5.94 \pm 1.25$ & $1.26 \pm 0.23$ & $0.04_{-0.02}^{+0.05}$ & $3.56 \pm 0.96$ \\
\hline NGC 4032 & $0.22 \pm 0.02$ & $0.05_{-0.01}^{+0.03}$ & $4.33 \pm 0.70$ & $1.35 \pm 0.18$ & $0.07_{-0.03}^{+0.06}$ & $3.01 \pm 0.58$ \\
\hline NGC 4214 & $1.23 \pm 0.08$ & $0.64_{-0.13}^{+0.22}$ & $1.91 \pm 0.24$ & $1.28 \pm 0.17$ & $1.03_{-0.37}^{+0.64}$ & $1.19 \pm 0.19$ \\
\hline NGC 4376 & $0.18 \pm 0.02$ & $0.03_{-0.01}^{+0.01}$ & $5.91 \pm 0.87$ & $1.41 \pm 0.16$ & $0.05_{-0.02}^{+0.04}$ & $3.89 \pm 0.80$ \\
\hline NGC 4449 & $1.31 \pm 0.08$ & $0.89_{-0.17}^{+0.21}$ & $1.46 \pm 0.14$ & $1.55 \pm 0.16$ & $1.18_{-0.47}^{+0.44}$ & $1.11 \pm 0.16$ \\
\hline NGC 4630 & $0.19 \pm 0.02$ & $0.10_{-0.02}^{+0.03}$ & $1.94 \pm 0.25$ & $1.46 \pm 0.16$ & $0.13_{-0.06}^{+0.05}$ & $1.44 \pm 0.23$ \\
\hline NGC 7694 & $0.19 \pm 0.02$ & $0.04_{-0.01}^{+0.01}$ & $4.39 \pm 0.73$ & $1.37 \pm 0.16$ & $0.06_{-0.03}^{+0.05}$ & $2.94 \pm 0.59$ \\
\hline UGC 04305 & $0.26 \pm 0.02$ & $0.07_{-0.06}^{+0.24}$ & $3.48 \pm 1.60$ & $1.21 \pm 0.31$ & $0.12_{-0.11}^{+0.63}$ & $2.09 \pm 1.29$ \\
\hline UGC 05720 & $0.05 \pm 0.02$ & $0.04_{-0.01}^{+0.02}$ & $1.29 \pm 0.43$ & $1.60 \pm 0.22$ & $0.05_{-0.03}^{+0.04}$ & $1.05 \pm 0.40$ \\
\hline
\end{tabular}

Note. Results of the SED fits using the fluxes in the wavelength range from $22-500 \mu \mathrm{m}$. The $850 \mu \mathrm{m}$ observed fluxes in this table have been subtracted for the contribution of non-dust components (CO(3-2) line emission, free-free and synchrotron emission) for our sample except for NGC $1427 \mathrm{~A}$. The $850 \mu \mathrm{m}$ observed flux of NGC 1427A has been only corrected for CO(3-2) line emission (see Appendix A). The uncertainty on the $850 \mu$ m observed-to-modeled flux $\left(\frac{850 \text { obs corrected }}{850 \text { modeled }}\right)$ combines the uncertainty from modeled flux (the standard deviation of posterior distributions of modeled flux) and the uncertainty of the observed flux $\left(F_{\nu}(850 \mu \mathrm{m})\right.$ ). 
Appendix C

Cold Dust Parameters Derived from the SED Fitting

Table 6 lists the fitting results of cold dust parameters $\left(\beta_{\mathrm{c}}, T_{\mathrm{c}}\right.$, and $\left.M_{\mathrm{c}}\right)$ derived from SED fits with data ranging from 22-160 $\mu \mathrm{m}, 22-250 \mu \mathrm{m}, 22-350 \mu \mathrm{m}, 22-500 \mu \mathrm{m}$, and $22-850 \mu \mathrm{m}$, respectively, included in the fitting program using the TMBB model with $\beta_{\mathrm{w}}=2$ and $\beta_{\mathrm{c}}=2$ or $\beta_{\mathrm{c}}$ as free and applying hierarchical Bayesian techniques.

Table 6

The Fitting Results of Cold Dust Parameters Derived from the SED Fits with Different Data Coverage

\begin{tabular}{|c|c|c|c|c|c|c|c|c|c|c|c|c|c|c|c|}
\hline \multirow{2}{*}{ Galaxy } & \multicolumn{3}{|c|}{$160 \mu \mathrm{m}$} & \multicolumn{3}{|c|}{$250 \mu \mathrm{m}$} & \multicolumn{3}{|c|}{$350 \mu \mathrm{m}$} & \multicolumn{3}{|c|}{$500 \mu \mathrm{m}$} & \multicolumn{3}{|c|}{$850 \mu \mathrm{m}$} \\
\hline & $\beta_{\mathrm{c}}$ & $T_{\mathrm{c}}(\mathrm{K})$ & $M_{\mathrm{c}}\left(\log M_{\odot}\right)$ & $\beta_{\mathrm{c}}$ & $T_{\mathrm{c}}(\mathrm{K})$ & $M_{\mathrm{c}}\left(\log M_{\odot}\right)$ & $\beta_{\mathrm{c}}$ & $T_{\mathrm{c}}(\mathrm{K})$ & $M_{\mathrm{c}}\left(\log M_{\odot}\right)$ & $\beta_{\mathrm{c}}$ & $T_{\mathrm{c}}(\mathrm{K})$ & $M_{\mathrm{c}}\left(\log M_{\odot}\right)$ & $\beta_{\mathrm{c}}$ & $T_{\mathrm{c}}(\mathrm{K})$ & $M_{\mathrm{c}}\left(\log M_{\odot}\right)$ \\
\hline \multirow[t]{2}{*}{ IC 0010} & 2 & $21.5 \pm 2.5$ & $6.01 \pm 0.34$ & 2 & $21.0 \pm 1.5$ & $6.07 \pm 0.21$ & 2 & $20.4 \pm 1.3$ & $6.13 \pm 0.19$ & 2 & $19.6 \pm 1.4$ & $6.24 \pm 0.21$ & 2 & $18.1 \pm 1.2$ & $6.38 \pm 0.13$ \\
\hline & $1.0 \pm 0.6$ & $26.4 \pm 4.9$ & $6.19 \pm 0.31$ & $1.3 \pm 0.5$ & $26.3 \pm 3.4$ & $6.04 \pm 0.26$ & $1.3 \pm 0.3$ & $25.0 \pm 2.1$ & $6.11 \pm 0.23$ & $1.3 \pm 0.2$ & $25.2 \pm 2.0$ & $6.09 \pm 0.19$ & $1.0 \pm 0.2$ & $30.3 \pm 2.8$ & $5.84 \pm 0.12$ \\
\hline \multirow[t]{2}{*}{ IC 3268} & 2 & $23.3 \pm 1.4$ & $6.95 \pm 0.15$ & 2 & $22.2 \pm 1.0$ & $7.09 \pm 0.09$ & 2 & $21.7 \pm 0.8$ & $7.15 \pm 0.08$ & 2 & $21.1 \pm 0.8$ & $7.20 \pm 0.07$ & 2 & $20.0 \pm 1.2$ & $7.30 \pm 0.09$ \\
\hline & $1.3 \pm 0.8$ & $27.9 \pm 4.8$ & $7.03 \pm 0.22$ & $1.4 \pm 0.4$ & $26.4 \pm 3.1$ & $7.07 \pm 0.09$ & $1.5 \pm 0.3$ & $25.3 \pm 1.9$ & $7.10 \pm 0.07$ & $1.4 \pm 0.2$ & $25.7 \pm 1.6$ & $7.13 \pm 0.06$ & $0.6 \pm 0.2$ & $33.4 \pm 3.0$ & $7.11 \pm 0.06$ \\
\hline \multirow[t]{2}{*}{ IC 3476} & 2 & $21.5 \pm 1.4$ & $6.55 \pm 0.16$ & 2 & $20.8 \pm 0.7$ & $6.65 \pm 0.07$ & 2 & $20.3 \pm 0.7$ & $6.69 \pm 0.06$ & 2 & $19.7 \pm 0.6$ & $6.75 \pm 0.05$ & 2 & $18.2 \pm 0.8$ & $6.87 \pm 0.06$ \\
\hline & $1.0 \pm 0.7$ & $26.2 \pm 4.6$ & $6.68 \pm 0.17$ & $1.3 \pm 0.5$ & $25.3 \pm 3.2$ & $6.63 \pm 0.07$ & $1.4 \pm 0.2$ & $24.5 \pm 1.8$ & $6.63 \pm 0.05$ & $1.4 \pm 0.2$ & $24.7 \pm 1.5$ & $6.63 \pm 0.05$ & $1.0 \pm 0.2$ & $28.4 \pm 1.9$ & $6.57 \pm 0.05$ \\
\hline \multirow[t]{2}{*}{ NGC 1427A } & 2 & $20.9 \pm 2.1$ & $6.33 \pm 0.31$ & 2 & $20.3 \pm 1.1$ & $6.41 \pm 0.12$ & 2 & $19.8 \pm 1.1$ & $6.49 \pm 0.10$ & 2 & $19.1 \pm 1.2$ & $6.55 \pm 0.10$ & 2 & $9.9 \pm 1.2$ & $7.63 \pm 0.18$ \\
\hline & $1.0 \pm 0.7$ & $25.0 \pm 4.6$ & $6.46 \pm 0.29$ & $1.2 \pm 0.6$ & $24.9 \pm 3.2$ & $6.42 \pm 0.12$ & $1.3 \pm 0.3$ & $24.2 \pm 2.0$ & $6.44 \pm 0.09$ & $1.3 \pm 0.2$ & $24.1 \pm 1.8$ & $6.45 \pm 0.09$ & $0.1 \pm 0.1$ & $31.5 \pm 2.2$ & $6.50 \pm 0.06$ \\
\hline \multirow[t]{2}{*}{ NGC 4032} & 2 & $22.5 \pm 1.6$ & $6.77 \pm 0.25$ & 2 & $21.3 \pm 1.1$ & $7.18 \pm 0.09$ & 2 & $20.7 \pm 1.1$ & $7.25 \pm 0.09$ & 2 & $19.6 \pm 1.3$ & $7.35 \pm 0.10$ & 2 & $12.1 \pm 0.9$ & $8.07 \pm 0.11$ \\
\hline & $1.1 \pm 0.7$ & $27.2 \pm 4.9$ & $6.88 \pm 0.26$ & $1.3 \pm 0.5$ & $25.9 \pm 3.6$ & $7.18 \pm 0.09$ & $1.4 \pm 0.2$ & $24.8 \pm 1.9$ & $7.20 \pm 0.07$ & $1.4 \pm 0.2$ & $24.9 \pm 1.7$ & $7.23 \pm 0.06$ & $0.3 \pm 0.2$ & $34.0 \pm 2.9$ & $7.18 \pm 0.06$ \\
\hline \multirow[t]{2}{*}{ NGC 4214} & 2 & $21.6 \pm 1.8$ & $6.31 \pm 0.19$ & 2 & $21.2 \pm 1.0$ & $6.35 \pm 0.08$ & 2 & $20.3 \pm 0.9$ & $6.43 \pm 0.07$ & 2 & $19.2 \pm 1.0$ & $6.53 \pm 0.07$ & 2 & $14.9 \pm 0.7$ & $6.91 \pm 0.06$ \\
\hline & $1.0 \pm 0.6$ & $26.4 \pm 4.7$ & $6.44 \pm 0.18$ & $1.3 \pm 0.4$ & $25.9 \pm 3.3$ & $6.32 \pm 0.08$ & $1.4 \pm 0.3$ & $24.8 \pm 2.0$ & $6.36 \pm 0.07$ & $1.3 \pm 0.2$ & $25.1 \pm 1.7$ & $6.40 \pm 0.06$ & $0.9 \pm 0.1$ & $30.0 \pm 2.2$ & $6.30 \pm 0.06$ \\
\hline \multirow[t]{2}{*}{ NGC 4376} & 2 & $22.6 \pm 1.2$ & $6.73 \pm 0.12$ & 2 & $22.0 \pm 0.9$ & $6.80 \pm 0.07$ & 2 & $21.5 \pm 0.9$ & $6.84 \pm 0.07$ & 2 & $20.9 \pm 0.8$ & $6.89 \pm 0.07$ & 2 & $19.4 \pm 1.1$ & $7.03 \pm 0.09$ \\
\hline & $1.2 \pm 0.7$ & $27.2 \pm 4.6$ & $6.81 \pm 0.19$ & $1.4 \pm 0.4$ & $26.2 \pm 3.6$ & $6.81 \pm 0.07$ & $1.5 \pm 0.2$ & $25.2 \pm 1.9$ & $6.82 \pm 0.06$ & $1.4 \pm 0.2$ & $25.7 \pm 1.6$ & $6.81 \pm 0.06$ & $0.5 \pm 0.2$ & $33.6 \pm 2.6$ & $6.83 \pm 0.05$ \\
\hline \multirow[t]{2}{*}{ NGC 4449} & 2 & $23.2 \pm 1.1$ & $6.82 \pm 0.10$ & 2 & $22.9 \pm 0.8$ & $6.83 \pm 0.06$ & 2 & $22.7 \pm 0.8$ & $6.85 \pm 0.05$ & 2 & $22.1 \pm 0.8$ & $6.89 \pm 0.05$ & 2 & $20.4 \pm 0.8$ & $7.02 \pm 0.04$ \\
\hline & $1.2 \pm 0.8$ & $28.0 \pm 4.8$ & $6.91 \pm 0.15$ & $1.5 \pm 0.4$ & $26.8 \pm 3.4$ & $6.81 \pm 0.06$ & $1.7 \pm 0.2$ & $25.8 \pm 2.1$ & $6.80 \pm 0.06$ & $1.6 \pm 0.2$ & $26.6 \pm 1.8$ & $6.80 \pm 0.05$ & $1.3 \pm 0.1$ & $29.6 \pm 1.5$ & $6.73 \pm 0.04$ \\
\hline \multirow[t]{2}{*}{ NGC 4630} & 2 & $22.5 \pm 1.2$ & $6.80 \pm 0.14$ & 2 & $20.7 \pm 0.8$ & $7.09 \pm 0.07$ & 2 & $20.5 \pm 0.7$ & $7.11 \pm 0.06$ & 2 & $20.1 \pm 0.6$ & $7.13 \pm 0.05$ & 2 & $18.6 \pm 0.7$ & $7.26 \pm 0.05$ \\
\hline & $1.1 \pm 0.7$ & $27.3 \pm 4.8$ & $6.89 \pm 0.19$ & $1.2 \pm 0.5$ & $25.5 \pm 3.8$ & $7.08 \pm 0.07$ & $1.4 \pm 0.2$ & $24.6 \pm 1.9$ & $7.05 \pm 0.06$ & $1.5 \pm 0.2$ & $24.8 \pm 1.7$ & $7.00 \pm 0.06$ & $1.1 \pm 0.1$ & $29.1 \pm 2.1$ & $6.93 \pm 0.06$ \\
\hline \multirow[t]{2}{*}{ NGC 7694} & $\ldots$ & $\ldots$ & $\ldots$ & 2 & $21.2 \pm 1.0$ & $6.56 \pm 0.08$ & 2 & $20.6 \pm 0.9$ & $6.62 \pm 0.07$ & 2 & $19.8 \pm 1.0$ & $6.68 \pm 0.07$ & 2 & $15.7 \pm 1.6$ & $7.00 \pm 0.14$ \\
\hline & $\ldots$ & $\ldots$ & $\ldots$ & $1.3 \pm 0.5$ & $25.9 \pm 3.3$ & $6.55 \pm 0.10$ & $1.4 \pm 0.3$ & $24.8 \pm 1.9$ & $6.55 \pm 0.07$ & $1.4 \pm 0.2$ & $25.1 \pm 1.6$ & $6.56 \pm 0.06$ & $0.6 \pm 0.2$ & $31.8 \pm 2.4$ & $6.52 \pm 0.05$ \\
\hline \multirow[t]{2}{*}{ UGC 04305} & $\ldots$ & $\ldots$ & $\ldots$ & 2 & $19.7 \pm 1.8$ & $5.63 \pm 0.23$ & 2 & $19.4 \pm 1.7$ & $5.67 \pm 0.23$ & 2 & $18.6 \pm 1.7$ & $5.76 \pm 0.23$ & 2 & $11.5 \pm 1.3$ & $6.66 \pm 0.12$ \\
\hline & $\ldots$ & $\ldots$ & $\ldots$ & $1.1 \pm 0.6$ & $24.7 \pm 3.7$ & $5.61 \pm 0.24$ & $1.3 \pm 0.4$ & $24.1 \pm 2.5$ & $5.60 \pm 0.22$ & $1.2 \pm 0.3$ & $24.1 \pm 2.6$ & $5.64 \pm 0.19$ & $0.5 \pm 0.2$ & $30.0 \pm 3.9$ & $5.66 \pm 0.12$ \\
\hline \multirow[t]{2}{*}{ UGC 05720} & 2 & $23.1 \pm 2.0$ & $7.05 \pm 0.19$ & 2 & $22.9 \pm 1.6$ & $7.00 \pm 0.11$ & 2 & $22.3 \pm 1.4$ & $7.03 \pm 0.08$ & 2 & $21.6 \pm 1.5$ & $7.07 \pm 0.08$ & 2 & $22.0 \pm 1.6$ & $7.06 \pm 0.09$ \\
\hline & $1.2 \pm 0.8$ & $28.0 \pm 5.5$ & $7.14 \pm 0.23$ & $1.6 \pm 0.4$ & $26.7 \pm 3.9$ & $6.95 \pm 0.10$ & $1.8 \pm 0.3$ & $25.6 \pm 2.7$ & $6.93 \pm 0.08$ & $1.6 \pm 0.2$ & $26.6 \pm 2.6$ & $6.96 \pm 0.08$ & $1.5 \pm 0.2$ & $30.6 \pm 3.0$ & $6.86 \pm 0.07$ \\
\hline
\end{tabular}

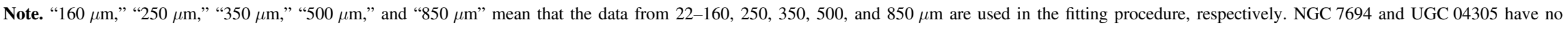
observation at $160 \mu \mathrm{m}$. 


\section{Appendix D \\ The Global SEDs of Sample}

Figure 10 shows the global SEDs of the remaining 11 galaxies obtained using the TMBB model $\left(\beta_{\mathrm{w}}=2\right.$ and $\beta_{\mathrm{c}}=2$ or $\beta_{\mathrm{c}}$ as free) with a hierarchical Bayesian technique to extrapolate 350,500 , and $850 \mu \mathrm{m}$ flux in the data ranges of $22-250 \mu \mathrm{m}, 22-350 \mu \mathrm{m}$, and $22-500 \mu \mathrm{m}$, respectively. Figure 11 shows the global SEDs of our sample with data ranging from $22-850 \mu \mathrm{m}$ using the same technique.
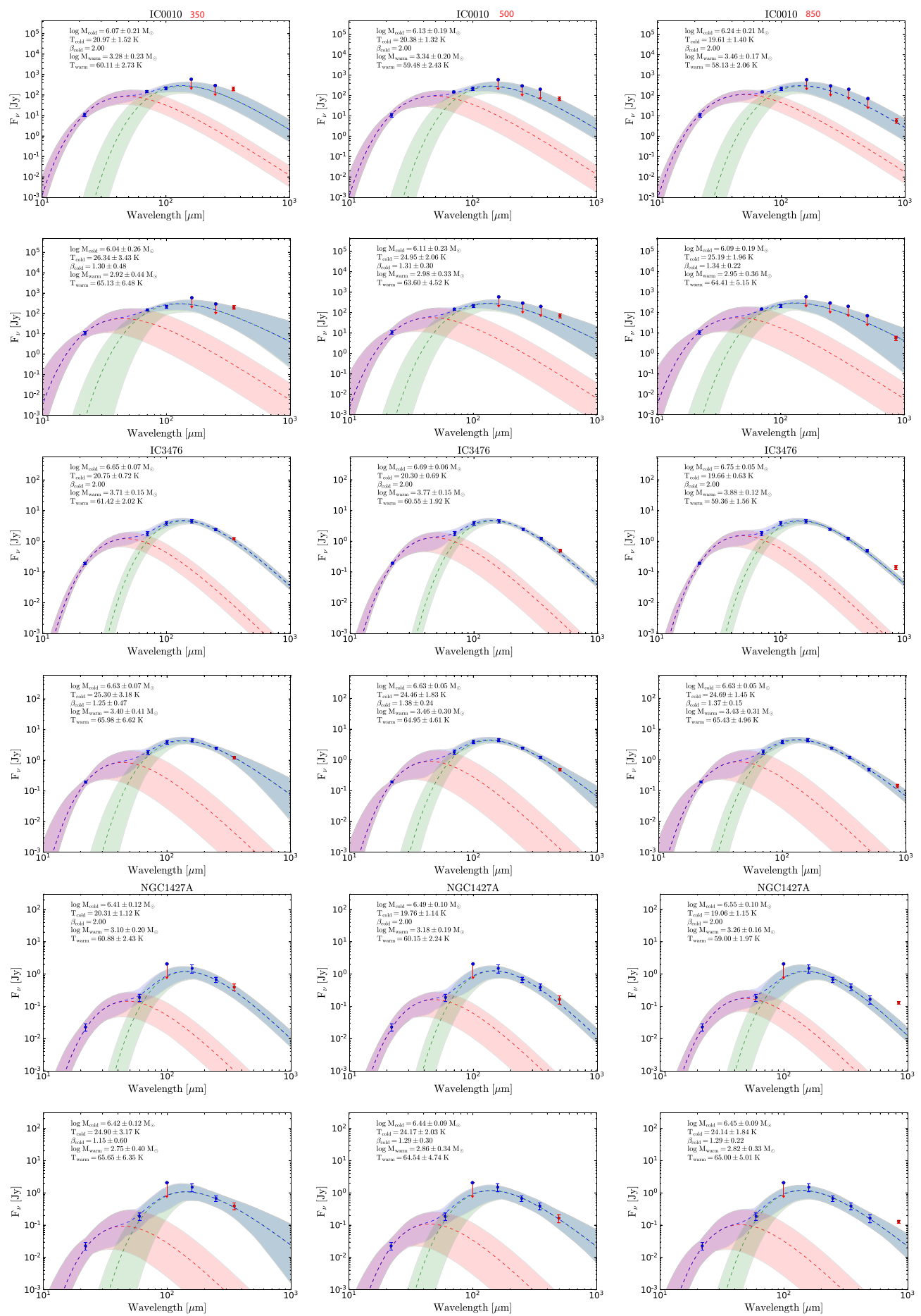

Figure 10. The global SEDs of the remaining 11 galaxies obtained using the TMBB model ( $\beta_{\mathrm{w}}=2$ and $\beta_{\mathrm{c}}=2$ or $\beta_{\mathrm{c}}$ as free) with a hierarchical Bayesian technique. For every galaxy, the first line of panels shows the SED fits in the case of $\beta_{\mathrm{c}}=2$ and the second line of panels shows the SED fits in the case of $\beta_{\mathrm{c}}$ as free. From left to right, the SEDs is to predict $350 \mu \mathrm{m}$ flux using the data ranging from $22-250 \mu \mathrm{m}$ to predict $500 \mu \mathrm{m}$ flux using the data ranging from $22-350 \mu \mathrm{m}$ and to predict $850 \mu \mathrm{m}$ flux using the data ranging from $22-500 \mu \mathrm{m}$, respectively. The warm component ( $\beta_{\mathrm{w}}$ fixed to 2$)$ is overlaid in red and the cold component $\left(\beta_{\mathrm{c}}\right.$ fixed to 2 or free) is in green. The blue area represents the sum of warm component and cold component. The shadow area shows the lower and upper $1 \sigma$ uncertainties on the SED models. WISE, Herschel, or Planck data (included in the fitting procedures) are overlaid with blue solid circles. The red solid circles (not included in the fitting) represent the observations at 350 , 500 , or $850 \mu \mathrm{m}$ shown in the panels from left to right, where the observed Planck flux densities at $850 \mu \mathrm{m}$ have been corrected for $\mathrm{CO}(3-2)$ line emission, free-free and synchrotron emission except for NGC 1427A. For NGC 1427A, the $850 \mu \mathrm{m}$ flux density has been only corrected for CO(3-2) line emission. 

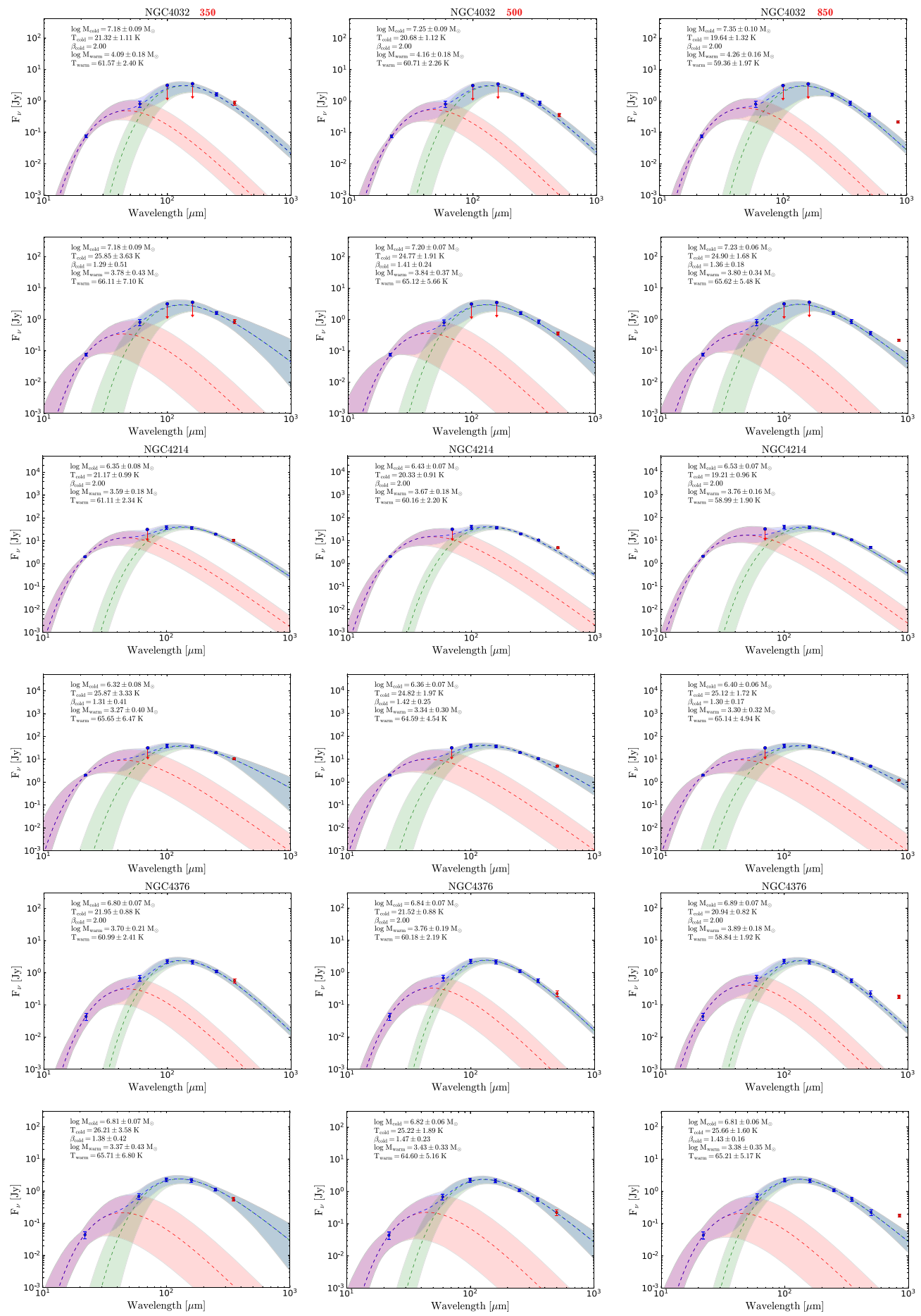

Figure 10. (Continued.) 

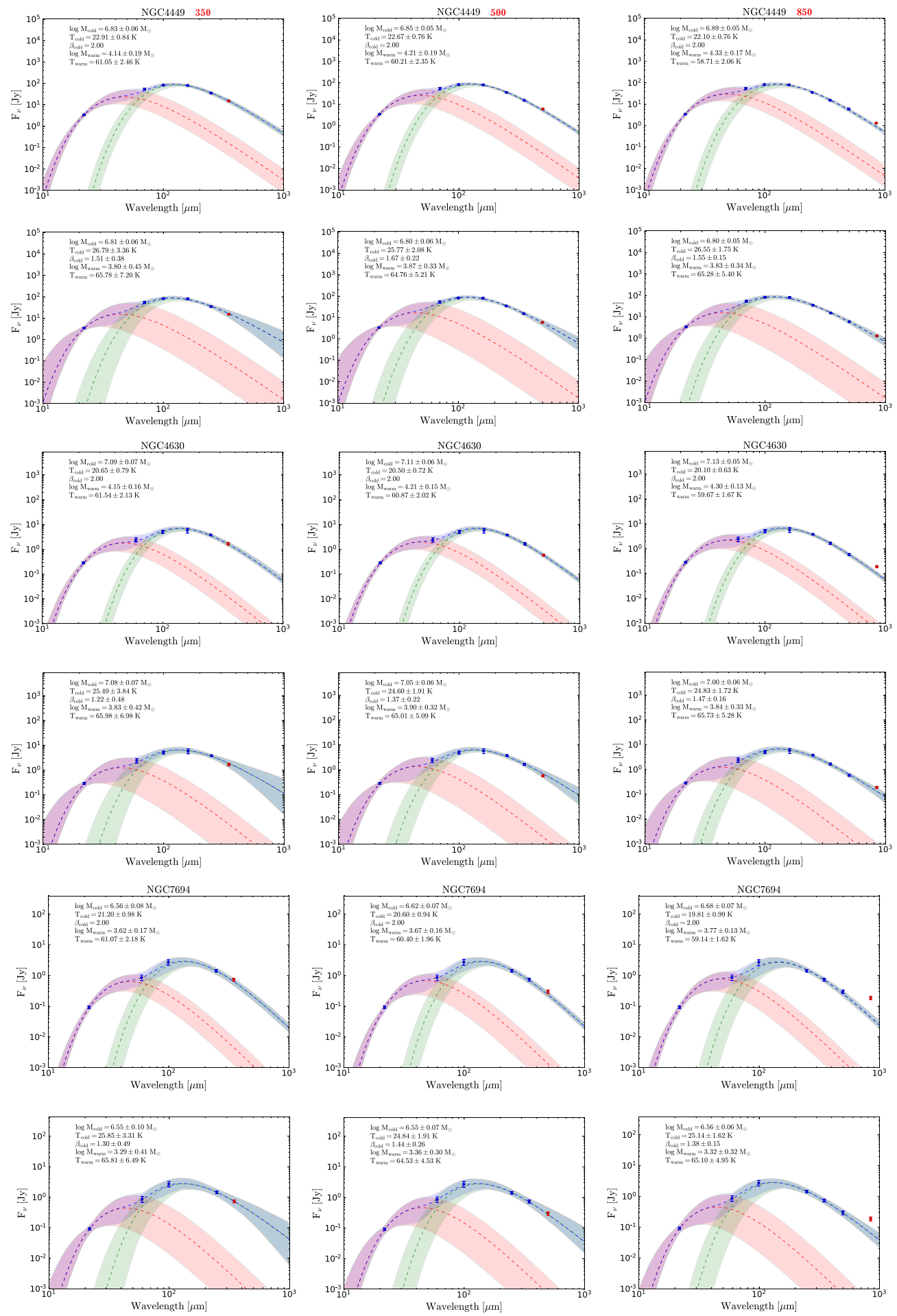

Figure 10. (Continued.) 

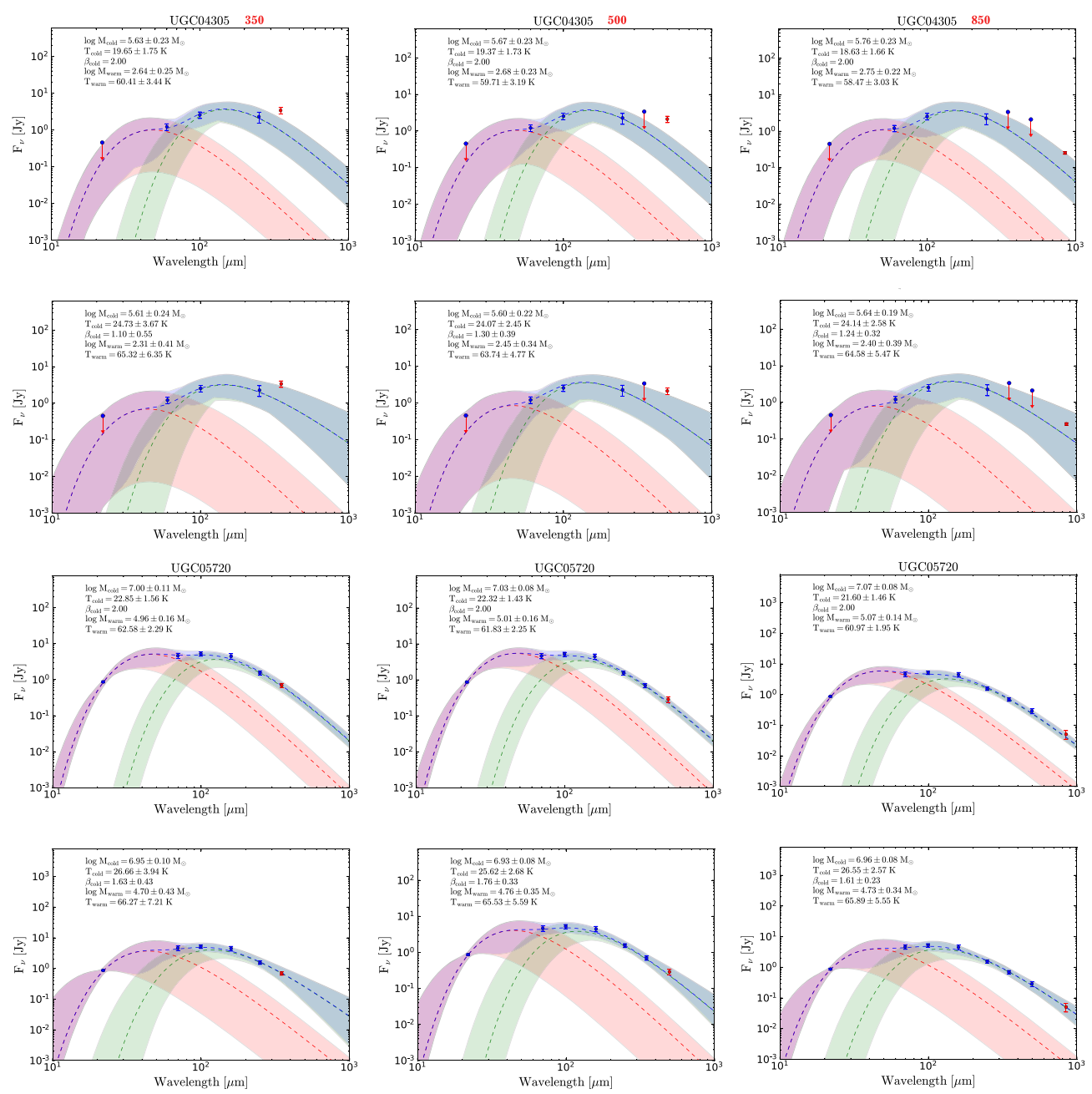

Figure 10. (Continued.) 

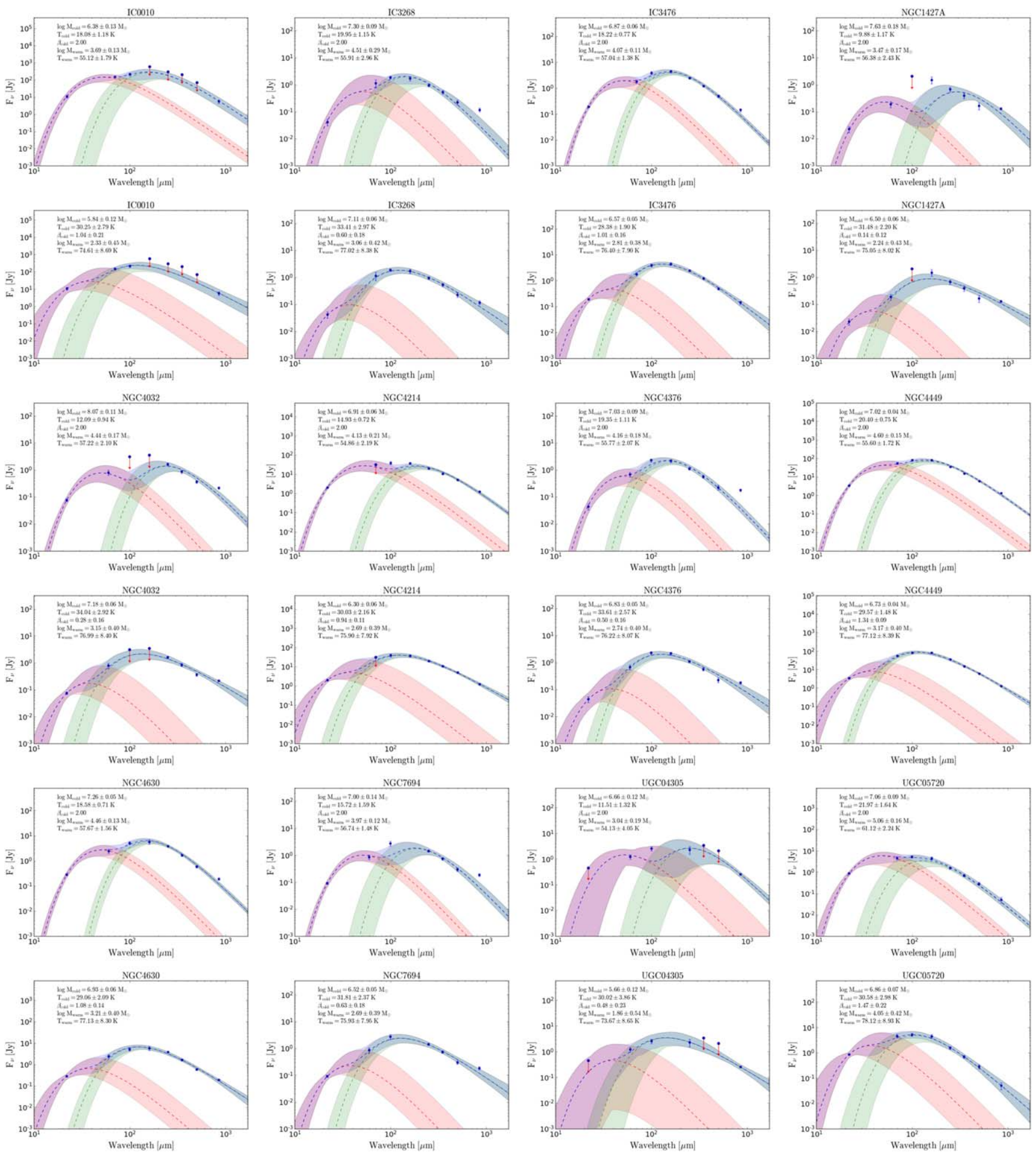

Figure 11. The global SEDs of our sample fitted with data from $22-850 \mu \mathrm{m}$ obtained by the same technique as Figure 10 . For the convention on colors and symbols, see Figure 10. The $850 \mu \mathrm{m}$ flux densities have been corrected for $\mathrm{CO}(3-2)$ line emission, free-free and synchrotron emission except for NGC 1427A. The 850 $\mu \mathrm{m}$ flux density of NGC 1427A has been only corrected for $\mathrm{CO}(3-2)$ line emission.

\section{ORCID iDs}

Zhengxue Chang (1) https://orcid.org/0000-0002-5981-7846 Jianjun Zhou (i) https://orcid.org/0000-0003-0356-818X Isabella Lamperti (1) https://orcid.org/0000-0003-3336-5498 Yuxin He (i) https://orcid.org/0000-0002-8760-8988 Jianjie Qiu (i) https://orcid.org/0000-0002-9829-8655 Xindi Tang (i) https://orcid.org/0000-0002-4154-4309
Gang Wu (ํ) https://orcid.org/0000-0003-0933-7112

Minhua Zhou (1) https://orcid.org/0000-0003-1213-2011

\section{References}

Accurso, G., Saintonge, A., Catinella, B., et al. 2017, MNRAS, 470, 4750

Bot, C., Ysard, N., Paradis, D., et al. 2010, A\&A, 523, A20

Bourne, N., Maddox, S., Dunne, L., et al. 2012, MNRAS, 421, 3027 
Bullock, J. S., \& Boylan-Kolchin, M. 2017, ARA\&A, 55, 343

Carpenter, B., Gelman, A., Hoffman, M. D., et al. 2017, J. Stat. Softw., 76, 1

Casasola, V., Bianchi, S., De Vis, P., et al. 2020, A\&A, 633, A100

Casassus, S., Cabrera, G., Förster, F., et al. 2006, ApJ, 639, 951

Chang, Z., Zhou, J., Wilson, C. D., et al. 2020, ApJ, 900, 53

Chini, R., Kruegel, E., Lemke, R., \& Ward-Thompson, D. 1995, A\&A, 295,317

Clark, C., Verstocken, S., Bianchi, S., et al. 2018, A\&A, 609, A37

Clark, C. J., Schofield, S. P., Gomez, H. L., \& Davies, J. I. 2016, MNRAS, 459, 1646

Clemens, M. S., Negrello, M., De Zotti, G., et al. 2013, MNRAS, 433, 695

Condon, J. 1992, ARA\&A, 30, 575

Cortese, L., Fritz, J., Bianchi, S., et al. 2014, MNRAS, 440, 942

da Cunha, E., Eminian, C., Charlot, S., \& Blaizot, J. 2010, MNRAS, 403, 1894

Dale, D., Aniano, G., Engelbracht, C., et al. 2012, ApJ, 745, 95

Davies, J., Baes, M., Bianchi, S., et al. 2017, PASP, 129, 044102

de Oliveira-Costa, A., Tegmark, M., Davies, R., et al. 2004, ApJL, 606, L89

De Vis, P., Dunne, L., Maddox, S., et al. 2017, MNRAS, 464, 4680

De Vis, P., Jones, A., Viaene, S., et al. 2019, A\&A, 623, A5

Desert, F. X., Boulanger, F., \& Puget, J. L. 1990, A\&A, 500, 313

Désert, F. X., Macías-Pérez, J. F., Mayet, F., et al. 2008, A\&A, 481, 411

Dobler, G., \& Finkbeiner, D. P. 2008, ApJ, 680, 1235

Draine, B., \& Hensley, B. 2012, ApJ, 757, 103

Draine, B., \& Lazarian, A. 1998, ApJ, 508, 157

Draine, B., \& Li, A. 2007, ApJ, 657, 810

Dunne, L., \& Eales, S. A. 2001, MNRAS, 327, 697

Dupac, X., Bernard, J. P., Boudet, N., et al. 2003, A\&A, 404, L11

Finkbeiner, D. P., Langston, G. I., \& Minter, A. H. 2004, ApJ, 617, 350

Galametz, M., Albrecht, M., Kennicutt, R., et al. 2014, MNRAS, 439, 2542

Galametz, M., Kennicutt, R., Albrecht, M., et al. 2012, MNRAS, 425, 763

Galametz, M., Madden, S., Galliano, F., et al. 2011, A\&A, 532, A56

Galametz, M., Madden, S. C., Galliano, F., et al. 2010, A\&A, 518, L55

Galliano, F. 2018, MNRAS, 476, 1445

Galliano, F., Hony, S., Bernard, J. P., et al. 2011, A\&A, 536, A88

Galliano, F., Madden, S., Jones, A., et al. 2003, A\&A, 407, 159

Gordon, K., Galliano, F., Hony, S., et al. 2010, A\&A, 518, L89

Górski, K., Hivon, E., Banday, A., et al. 2005, ApJ, 622, 759

Hermelo, I., Lisenfeld, U., Relaño, M., et al. 2013, A\&A, 549, A70

Hermelo, I., Relaño, M., Lisenfeld, U., et al. 2016, A\&A, 590, A56
Izotov, Y., Guseva, N., Fricke, K., Krügel, E., \& Henkel, C. 2014, A\&A, 570, A97

Jiang, X.-J., Wang, Z., Gu, Q., Wang, J., \& Zhang, Z.-Y. 2015, ApJ, 799, 92 Juvela, M., Montillaud, J., Ysard, N., \& Lunttila, T. 2013, A\&A, 556, A63

Kelly, B. C., Shetty, R., Stutz, A. M., et al. 2012, ApJ, 752, 55

Klaas, U., Haas, M., Müller, S., et al. 2001, A\&A, 379, 823

Kong, X., Lian, J., Gao, Y., et al. 2019, in IAU Symp. 344, Dwarf Galaxies: From the Deep Universe to the Present, ed. K. B. McQuinn \& S. Stierwalt (Cambridge: Cambridge Univ. Press), 437

Krugel, E., Siebenmorgen, R., Zota, V., \& Chini, R. 1998, A\&A, 331, L9

Lamperti, I., Saintonge, A., De Looze, I., et al. 2019, MNRAS, 489, 4389

Lamperti, I., Saintonge, A., Koss, M., et al. 2020, ApJ, 889, 103

Li, A., \& Draine, B. 2001, ApJ, 554, 778

Lisenfeld, U., Israel, F., Stil, J., \& Sievers, A. 2002, A\&A, 382, 860

Mao, R.-Q., Schulz, A., Henkel, C., et al. 2010, ApJ, 724, 1336

Miville-Deschênes, M. A., Ysard, N., Lavabre, A., et al. 2008, A\&A, 490, 1093

Niklas, S., Klein, U., \& Wielebinski, R. 1997, A\&A, 322, 19

Planck Collaboration, Adam, R., Ade, P. A. R., et al. 2016a, A\&A, 594, A10

Planck Collaboration, Ade, P., Aghanim, N., et al. 2014, A\&A, 571, A28

Planck Collaboration, Ade, P. A. R., Aghanim, N., et al. 2016b, A\&A, 594, A26

Planck Collaboration, Aghanim, N., Akrami, Y., et al. 2020a, A\&A, 641, A3

Planck Collaboration, Aghanim, N., Akrami, Y., et al. 2020b, A\&A, 641, A1

Popescu, C. C., Tuffs, R. J., Völk, H. J., Pierini, D., \& Madore, B. F. 2002, ApJ, 567, 221

Reach, W., Dwek, E., Fixsen, D., et al. 1995, ApJ, 451, 188

Relaño, M., De Looze, I., Kennicutt, R. C., et al. 2018, A\&A, 613, A43

Rémy-Ruyer, A., Madden, S., Galliano, F., et al. 2013, A\&A, 557, A95

Rémy-Ruyer, A., Madden, S. C., Galliano, F., et al. 2015, A\&A, 582, A121

Shetty, R., Kauffmann, J., Schnee, S., \& Goodman, A. A. 2009a, ApJ, 696, 676

Shetty, R., Kauffmann, J., Schnee, S., Goodman, A. A., \& Ercolano, B. 2009b, ApJ, 696, 2234

Shi, Y., Wang, J., Zhang, Z.-Y., et al. 2016, NatCo, 7, 13789

Skibba, R. A., Engelbracht, C. W., Dale, D., et al. 2011, ApJ, 738, 89

Solomon, P., Downes, D., Radford, S., \& Barrett, J. 1997, ApJ, 478, 144

Stan Development Team 2018, PyStan: the Python interface to Stan, v2.17.1.0, https://pystan.readthedocs.io/en/latest/

Tibbs, C. T., Israel, F. P., Laureijs, R. J., et al. 2018, MNRAS, 477, 4968

Veneziani, M., Piacentini, F., Noriega-Crespo, A., et al. 2013, ApJ, 772, 56 